\title{
Analysis on Detection of Chalkiness for Myanmar Rice Using Image Processing
}

\author{
Thae Nu Wah ${ }^{1}$, Hla Myo Tun ${ }^{2}$ \\ ${ }^{1}$ Department of Electronic Engineering, Technological University (Thanlyin), Yangon, Myanmar \\ ${ }^{2}$ Department of Electronic Engineering, Yangon Technological University, Yangon, Myanmar
}

Email address:

thaenuwah.tnw@gmail.com (T. Nu Wah),hlamyotun.ytu@gmail.com (H. M. Tun)

\section{To cite this article:}

Thae Nu Wah, Hla Myo Tun. Analysis on Detection of Chalkiness for Myanmar Rice Using Image Processing. Machine Learning Research. Vol. 3, No. 2, 2018, pp. 33-48. doi: 10.11648/j.mlr.20180302.14

Received: August 12, 2018; Accepted: August 28, 2018; Published: September 25, 2018

\begin{abstract}
The paper presents the analysis on detection of chalkiness of Myanmar Rice using image processing with the help of MATLAB. Chalkiness is a major control in rice production because it is one of the key factors determining grain quality (appearance, processing, milling, storing, eating, and cooking quality) and price. Its reduction is a major goal, and the primary purpose of this study was to scrutinize the genetic basis of grain chalkiness. Recent researches have shown that elevated nighttime air temperatures (NTATs) could contribute to increased chalk and reduced milling quality. Machine vision has been used in a most application of grain classification to differentiate rice varieties based on special features such as shape, length, chalkiness, colour and internal damage of rice. There are many kinds of rice in Myanmar. Among them, the Enatha, KaungNyib, nurserySticky, Paw-San and Zee Yar are famous types of rice for daily usages in Myanmar. In this paper, the analysis has been emphasized on those kinds of rice with the help of image processing techniques. The detection method for rice chalkiness has been analysed on the various kinds of Myanmar rice such as Ematha (20\%) 1.0A, KaungNyin3, nurserySticky110, Paw-San C and zee yar10. The results show that the rice chalkiness distribution function based on area of interest (location) and is could be measured with chalkiness intensity in this paper.
\end{abstract}

Keywords: Detection Method, Chalkiness, Myanmar Rice, Digital Image Processing, Distribution Function

\section{Introduction}

Rice is one of the most important food crops worldwide and contributes to $95 \%$ of the total calorie intake for Myanmar people. With the improvement of living standards, there is an increasing demand for better grain quality [1-3]. Rice grain quality is a complex character with several components, including grain appearance and milling, eating, cooking, and nutritional qualities; appearance quality is mostly determined by grain shape and endosperm opacity (or chalkiness) [4]. Chalkiness is divided into white belly, white core (WC), and white back (WB) in rice grain, depending upon its location on or within the endosperm. It represents a major problem in rice production in many rice-producing areas of the world because chalkiness results in inferior milling, cooking, eating, and nutritional quality [5-11]. Thus, grain chalkiness determines grain quality and price, and its reduction is an important goal of artificial breeding in rice.
Chalky rice kernels generally result in lower head rice yield (HRY), a quality indicator that determines the market value of rice, because they tend to be weaker and more prone to breakage during milling than translucent kernels $[12,13]$. Warm nighttime air temperatures (NTATs) are reported to interfere with metabolic processes during developmental stages in rice, which may result in the formation of chalk. [14] reported that rice cultivars grown in controlledenvironment growth chambers showed different degrees of susceptibility to high NTATs with respect to chalkiness and further proposed that increased chalk formation in rice could be due to a reduction in the rates of enzymatic activity and physiological functioning during high-NTAT exposure.

The purpose of the image processing techniques is testing the quality of the rice grain. The quality of the rice grain is based on the several parameters. Such as grain colour, shape, and the size. Fragments of the grain are highly effective to the quality of the rice. Machine vision systems are used to identify the quality of the grain. Digital images and videos 
are the key sources of the machine vision systems. When the grains are mixing together machine vision system needs to identify contains the grains mixture.

\section{Background}

Rice is one of the primary food crops in the world. Actually, rice yield estimation is an important aspect of the agriculture research field. The quality of the most staple food crop of world which is Rice is determined by shape, size aroma, nutritional value and the texture of the grain kernels. Broken rice grains appear during husking and adulteration, so there is always a need for the quality of the rice. During each harvest year and at each location, rice samples of each cultivar were harvested in triplicate over a range of moisture contents (MCs). Chalk values were determined and averaged across all sample lots within each harvest year/location/cultivar combination. In brief, duplicate $100-\mathrm{g}$ rough rice samples from each harvest lot were de-hulled to produce brown rice. One hundred brown rice kernels from each sample were randomly selected and placed on a tray (152 $\mathrm{mm} \times 100 \mathrm{~mm} \times 20 \mathrm{~mm}$ ) made from $32-\mathrm{mm}$ thick, clear acrylic sheet, so that no single kernel touched another kernel. A digital image of kernels was created by placing the tray on the scanner of an image analysis system. Prior to analysis, the imaging system was configured to color-classify chalk by selecting and scanning a brown rice kernel considered to be completely chalky into the imaging system as a reference color for chalk. The imaging system measured and recorded the number of pixels representing the entire kernel area from the scanned images, as well as the number of pixels corresponding to those areas color-classified for chalk on a kernel. Percent chalk in a sample was determined as the ratio of the total chalky area (pixels) of the 100-kernel set to the total area of the kernels, multiplied by 100 .

Recent years, electronic technique has been applied to the modern agriculture research widely. Specially, the application of computer vision by the means of the digital image camera is quite popular [15]. To the best of our knowledge, no related literatures about the rice density calculation utilizing computer vision method have been presented.

Due to the limited capabilities of Human eye we propose a new technique using Image Processing for Physical Quality Detection. Rice grain Kernels are near about elliptical in shape and so possess an aspect ratio. Each rice variety has more or less the same aspect ratio to its grain kernels. With the variation of the rice variety and broken grains we observe a change in elliptical properties. Statistical Analysis on the grain properties makes the quality result using comparison with ideal variety results.

Image processing is a useful tool in application of agriculture, metallurgy, biotechnology, etc. Image processing being a non-destructive, economical, fast and accurate has been beneficial tool for analysis. Digital image analysis has also been used to evaluate the effect of moisture content on cereal grains by studying its effect on the physical appearance and kernel morphology.

\section{Implementation}

The implementation of analysis on rice grain chalkiness detection could be followed the step-by-step procedure of the flowchart of Figure 1 in this paper. In this flowchart, the rice image has to be read by MATLAB commands. After that the rice image could be flattened and segmented based on the chalkiness detection system. And then, the detected rice chalkiness could be generated as the chalkiness distribution function with area of interested from rice.

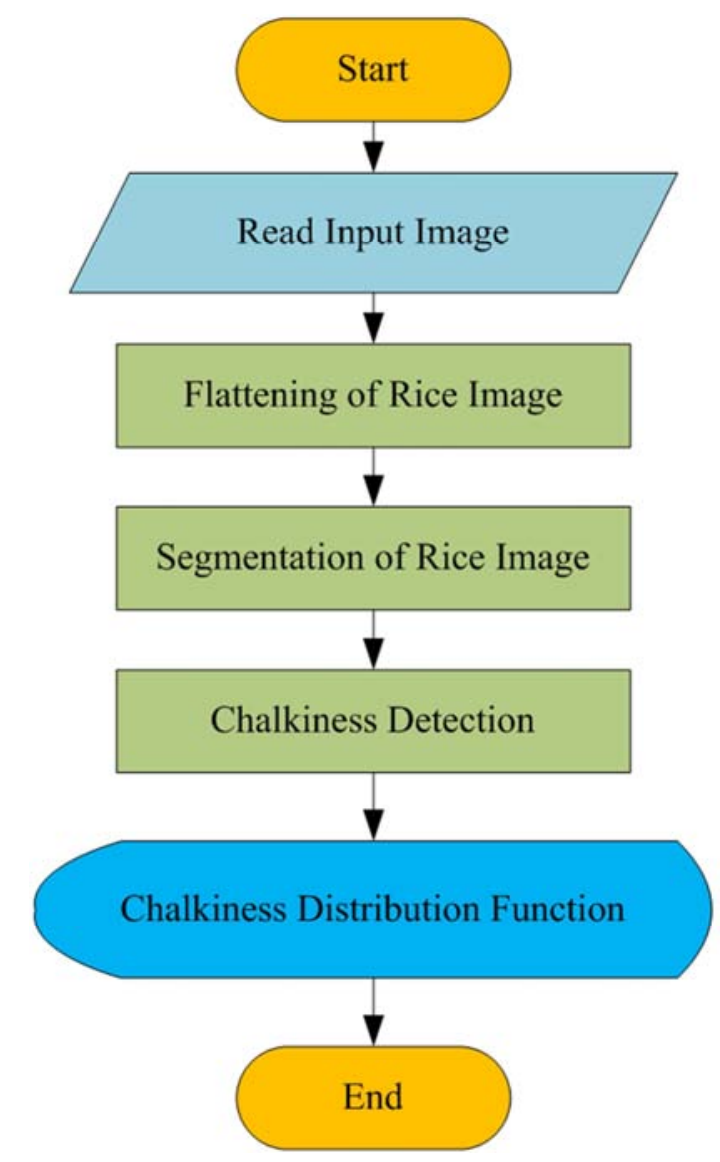

Figure 1. Flowchart of Implementation.

\subsection{Image Capture}

The images used in the program are obtained either with Digital Image Photography or Flat Bed Scanning. Both use a black background for its post-capturing works. For Flat Bed Scanning the rice were scattered in the glass panel of the flatbed scanner and a black background was used. For Digital Image Photography a fixed distanced between the objects and the camera was used for capturing the image. The images were converted into JPEG format for further processing. Two images are used. First image has the best finely selected rice grain which is used for reference and the second image is of the grain which is to be tested.

\subsection{Image Segmentation}

The following steps were carried out for processing. The captured images are converted to gray scale images. 
Backgrounds of the Images are subtracted from the images. The images are further processed with contrast adjustment. Images are converted into binary Images for performing further filtering.

Owing to elliptical shape of the rice grain kernels, the major axis, minor axis and aspect ratio of each grain can be determined. The images obtained after processing are segmented to obtain the physical properties which is their major axis and minor axis of each grain. The value of Major axis and Minor axis obtained is used to calculate the aspect ratio is used as a parametric value. Two separate one dimensional arrays are used for storing the values. First array is used for storing the parameter values of each grain of the reference sample and may be nomenclature as reference parametric array. The Second array is used to store the parameter values of each grain of the sample parametric array. The reference parametric array and sample parametric array are used for further statistical analysis.

\section{Simulation Results}

Based on the implemented step from the section 3, there are many kinds of Myanmar rice such as Ematha (20\%) 1.0A, KaungNyin3, nurserySticky110, Paw-San C and zee yar10. There are five steps to detect the rice grain chalkiness for those Myanmar rice. There are many screenshot results based on those five steps analysis like read the rice image, flattening, segmenting, detection of rice chalkiness and generate the chalkiness distribution function from those analysis. Figure 2 shows the screenshot result of read image of Ematha (20\%) 1.0A.jpg from the database. Figure 3 demonstrates the screenshot result of flatten condition for Ematha (20\%) 1.0A.jpg. Figure 4 mentions the screenshot result of threshold condition for Ematha (20\%) 1.0A.jpg. Figure 5 illustrates the screenshot result of chalkiness detection for Ematha (20\%) 1.0A.jpg. Figure 6 shows the chalkiness distribution function for Ematha (20\%) 1.0A.jpg. According to the distribution function, the area of 0 to 500 is decrease and the area of 500 to 1000 is a little bit higher.

Figure 7 mentions the screenshot result of read image of KaungNyin3.jpg from the database. Figure 8 illustrates the screenshot result of flatten condition for KaungNyin3.jpg. Figure 9 shows the screenshot result of threshold condition for KaungNyin3.jpg. Figure 10 demonstrates the screenshot result of chalkiness detection for KaungNyin3.jpg. Figure 11 points out the chalkiness distribution function for KaungNyin3.jpg. According to the distribution function, the area of 0 to 200 is the highest level and the area of 600 to 1000 is a Gaussian shape.

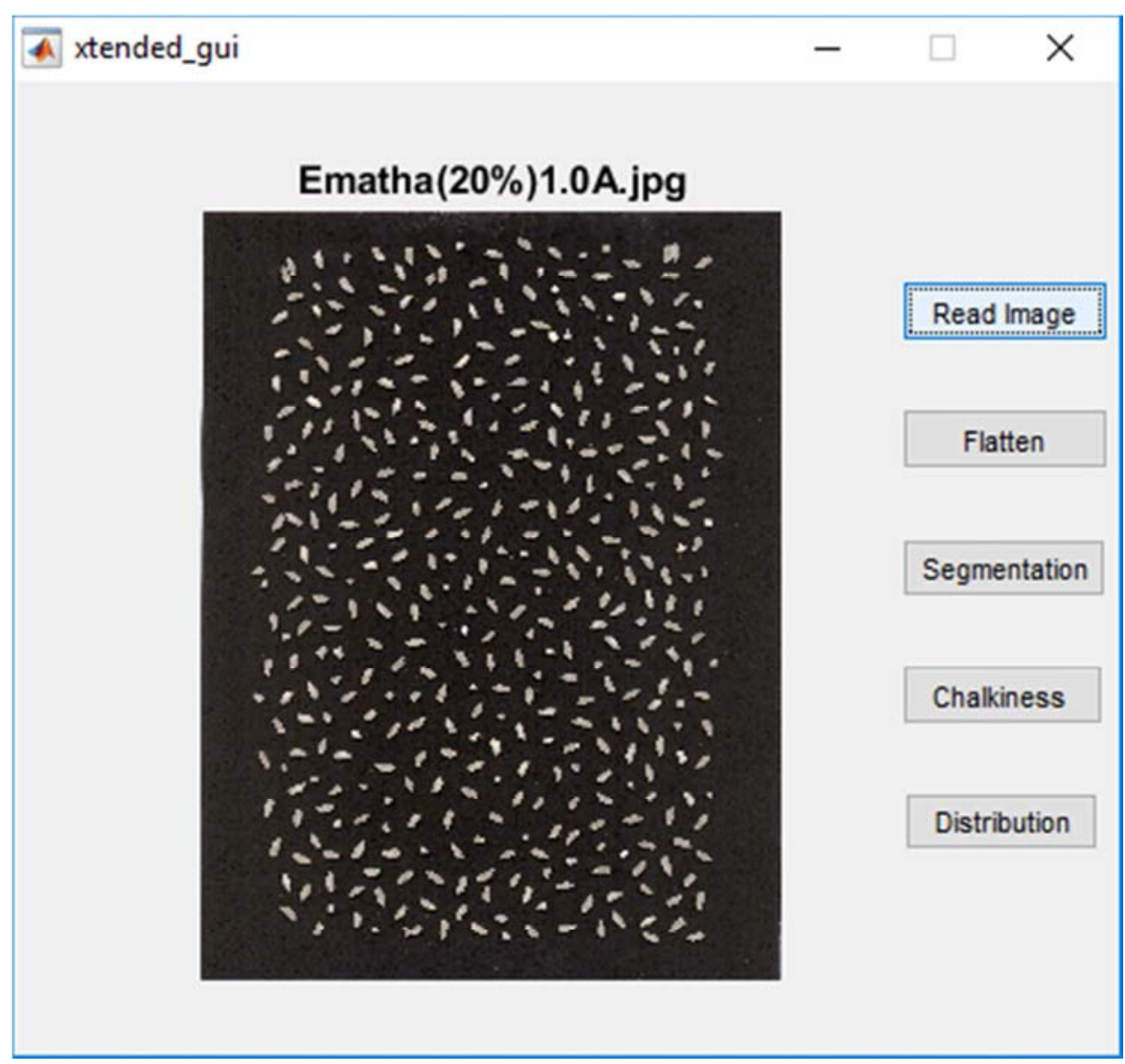

Figure 2. Screenshot Result of Read Image of Ematha (20\%) 1.0A.jpg from the Database. 


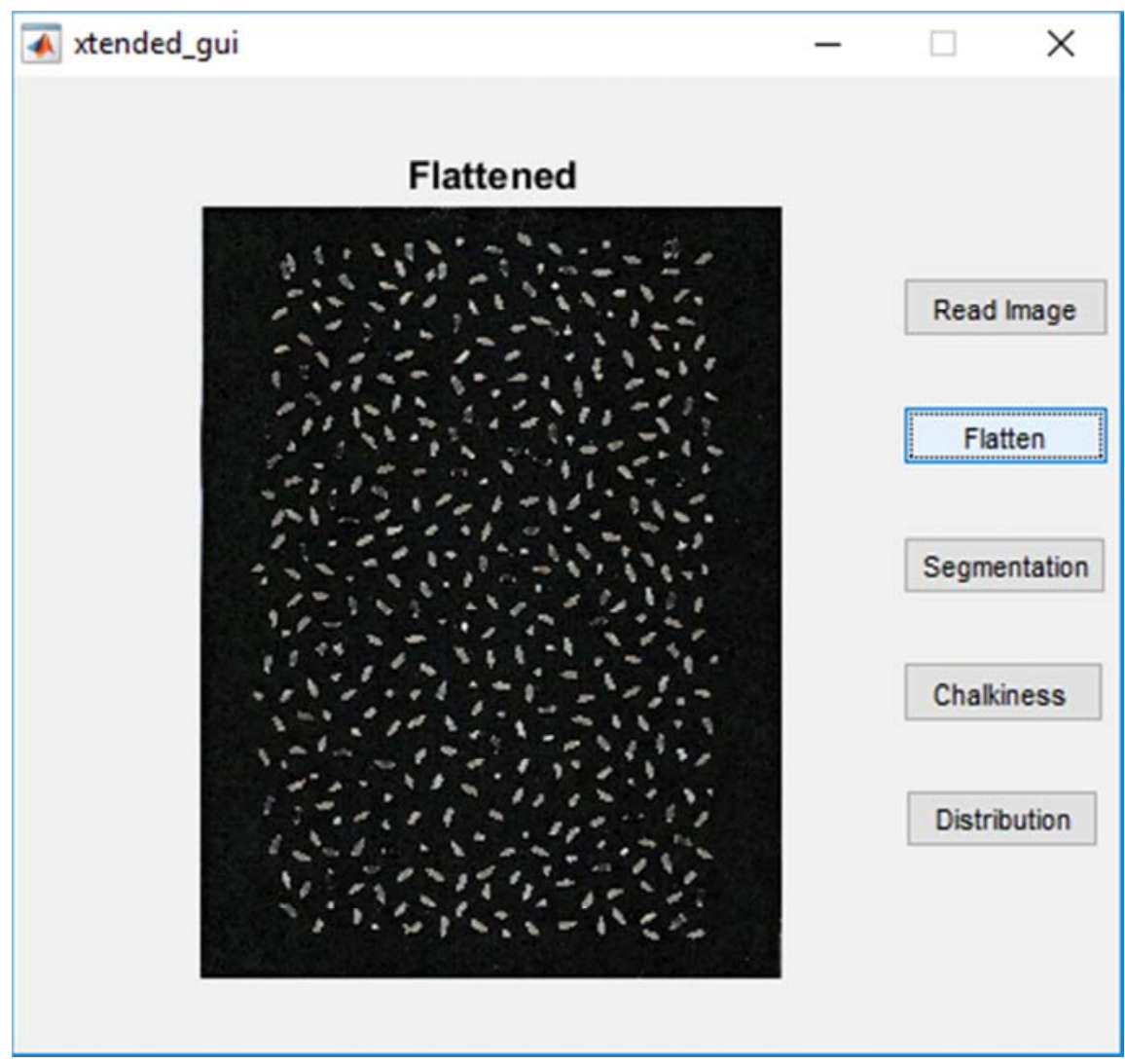

Figure 3. Screenshot Result of Flatten Condition for Ematha (20\%) 1.0A.jpg.

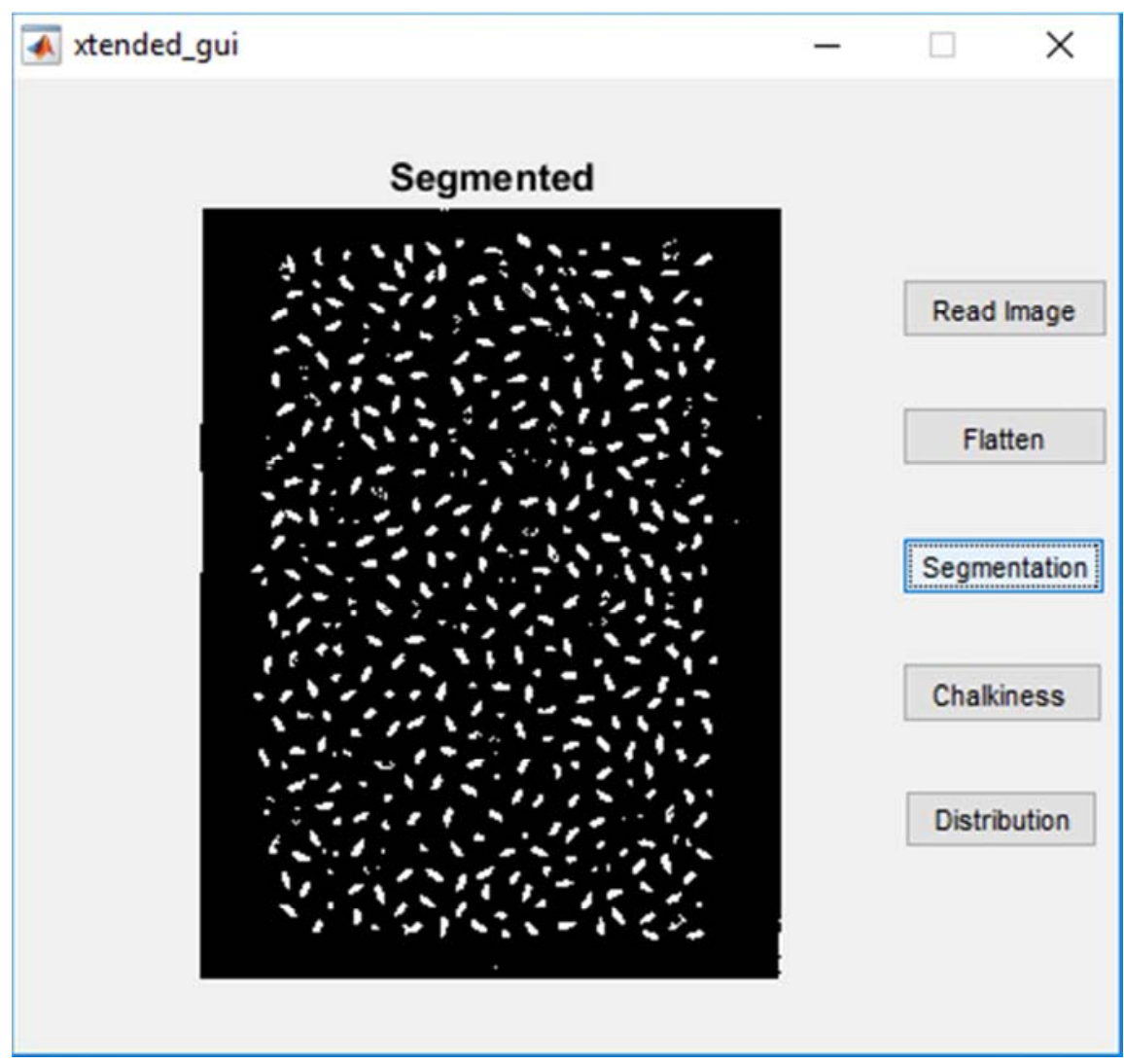

Figure 4. Screenshot Result of Threshold Condition for Ematha (20\%) 1.0A.jpg. 


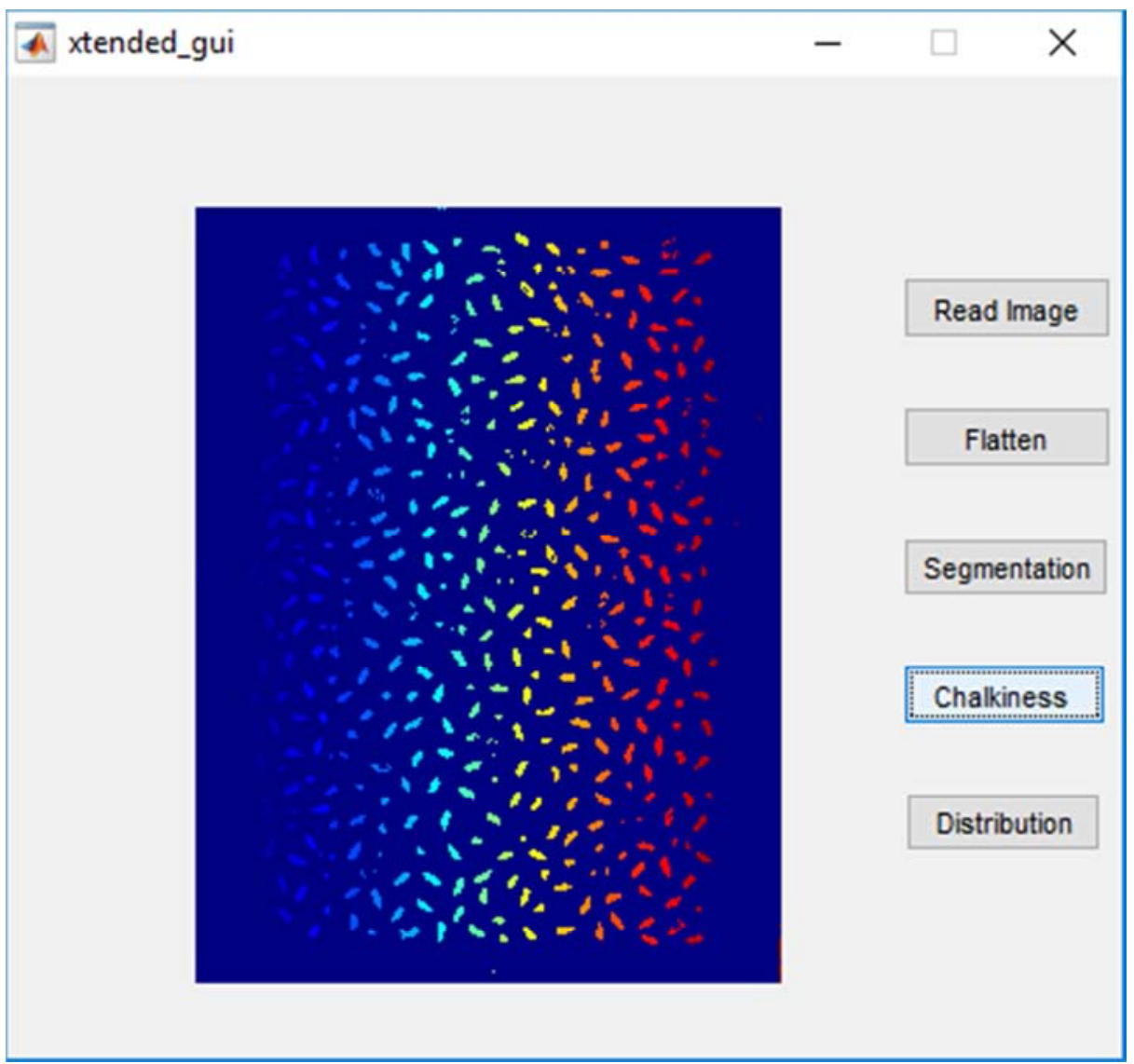

Figure 5. Screenshot Result of Chalkiness Detection for Ematha (20\%) 1.0A.jpg.

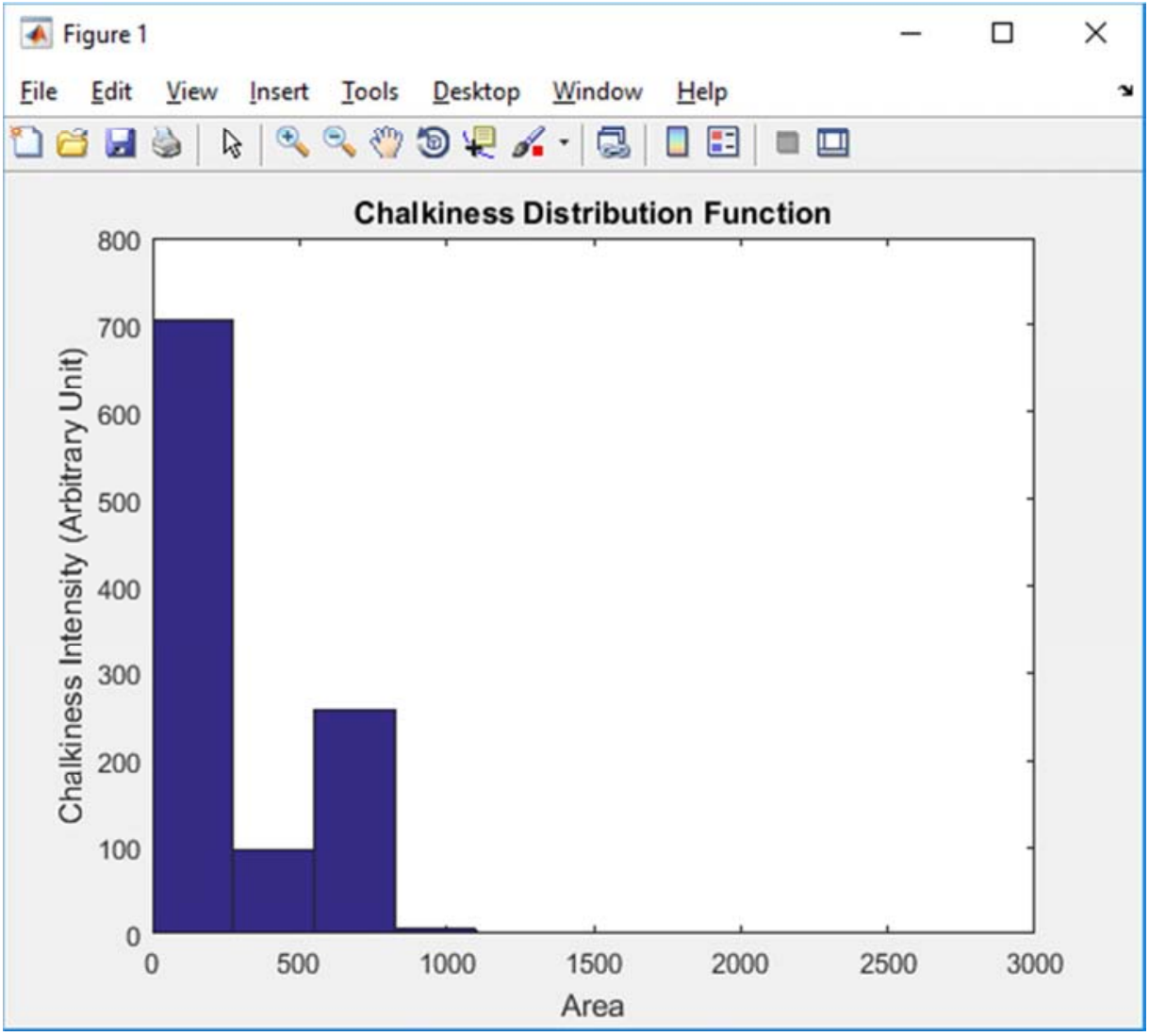

Figure 6. Chalkiness Distribution Function for Ematha (20\%) 1.0A.jpg. 


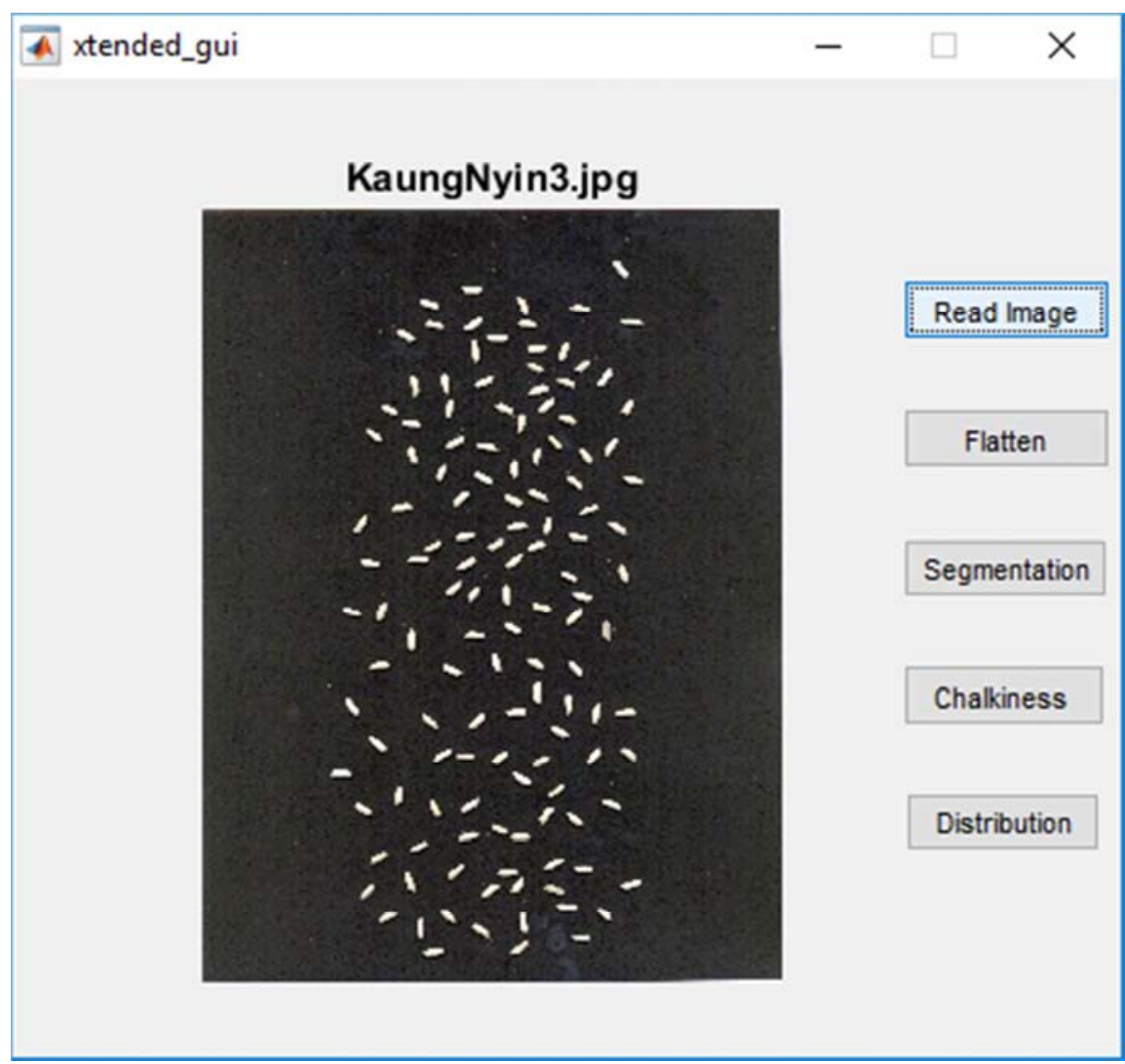

Figure 7. Screenshot Result of Read Image of KaungNyin3.jpg from the Database.

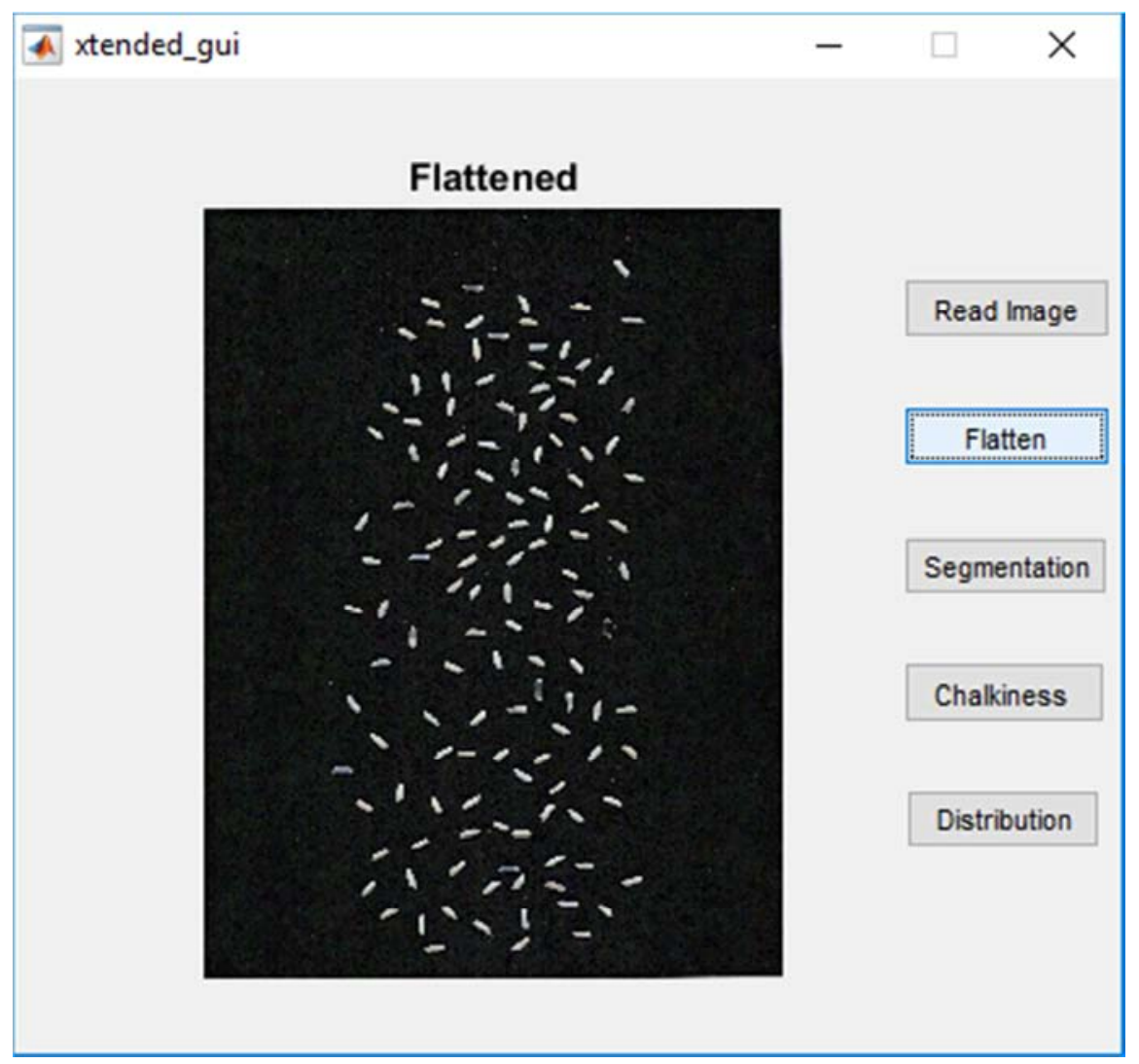

Figure 8. Screenshot Result of Flatten Condition for KaungNyin3.jpg 


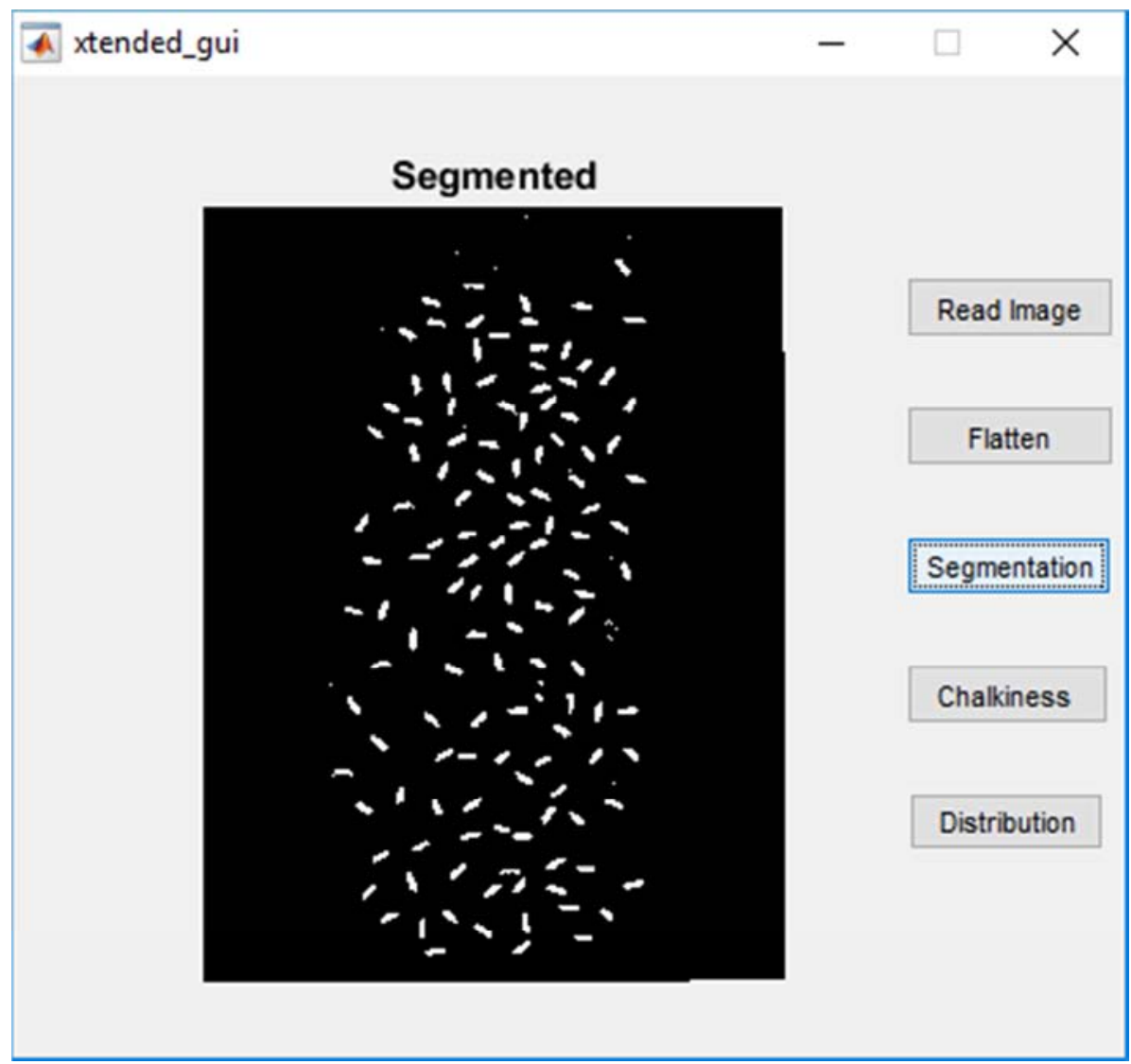

Figure 9. Screenshot Result of Threshold Condition for KaungNyin3.jpg.

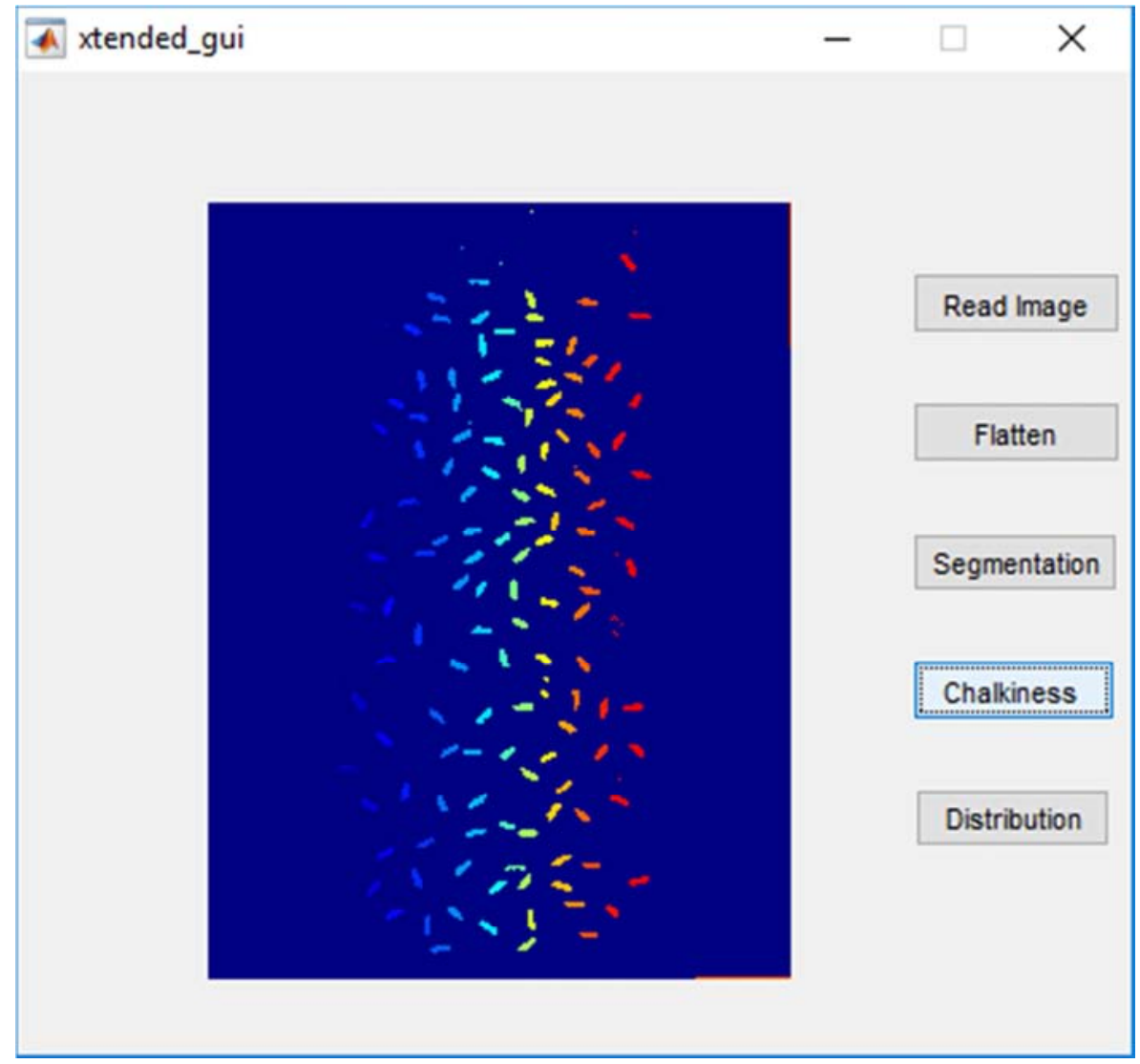

Figure 10. Screenshot Result of Chalkiness Detection for KaungNyin3.jpg. 


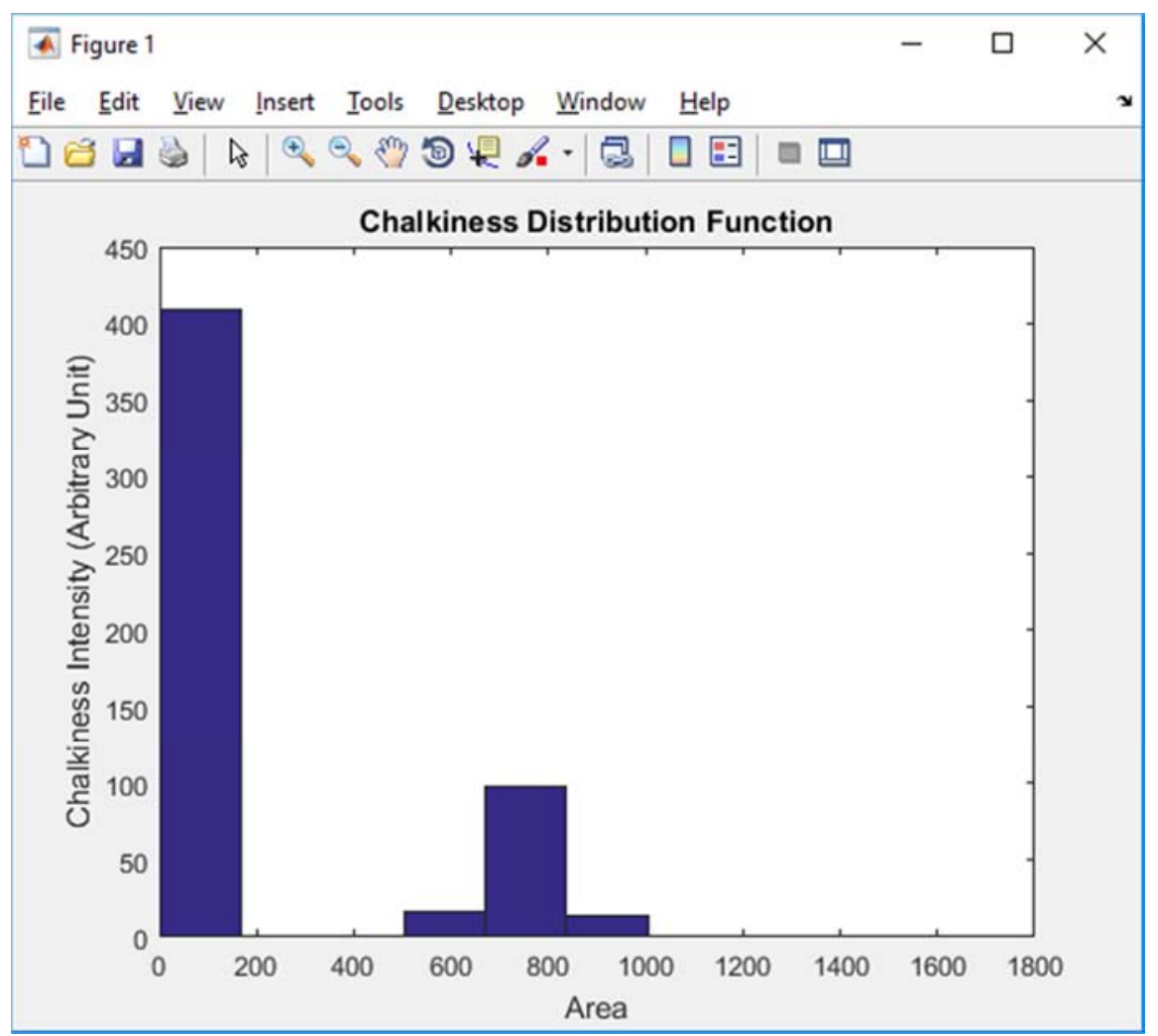

Figure 11. Chalkiness Distribution Function for KaungNyin3.jpg.

Figure 12 shows the screenshot result of read image of nurserySticky110.jpg from the database. Figure 13 mentions the screenshot result of flatten condition for nurserySticky110.jpg. Figure 14 illustrates the screenshot result of threshold condition for nurserySticky110.jpg. Figure
15 demonstrates the screenshot result of chalkiness detection for nurserySticky110.jpg. Figure 16 shows the chalkiness distribution function for nurserySticky110.jpg. According to the distribution function, the area of 0 to 200 is the highest level and the area of 200 to 1000 is a Gaussian shape.

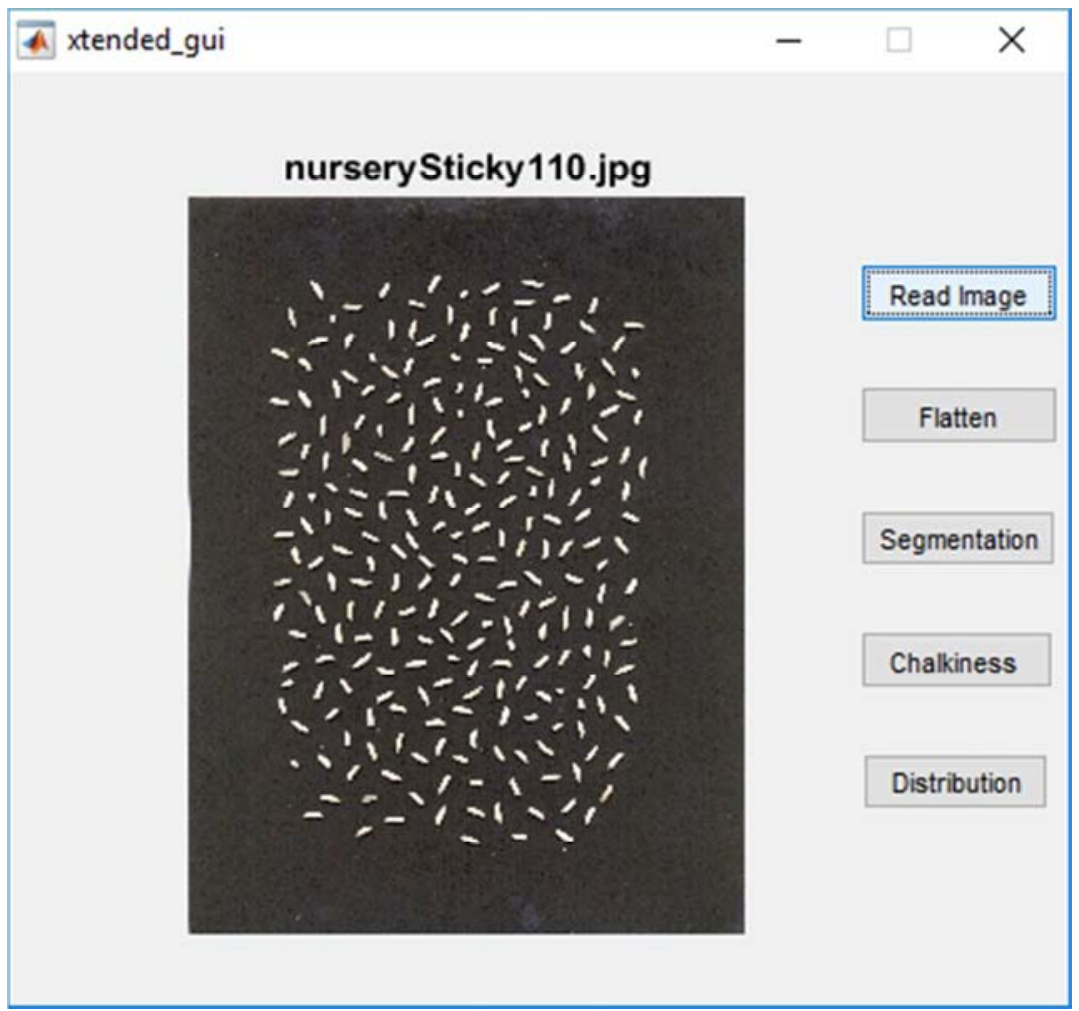

Figure 12. Screenshot Result of Read Image of nurserySticky110.jpg from the Database. 


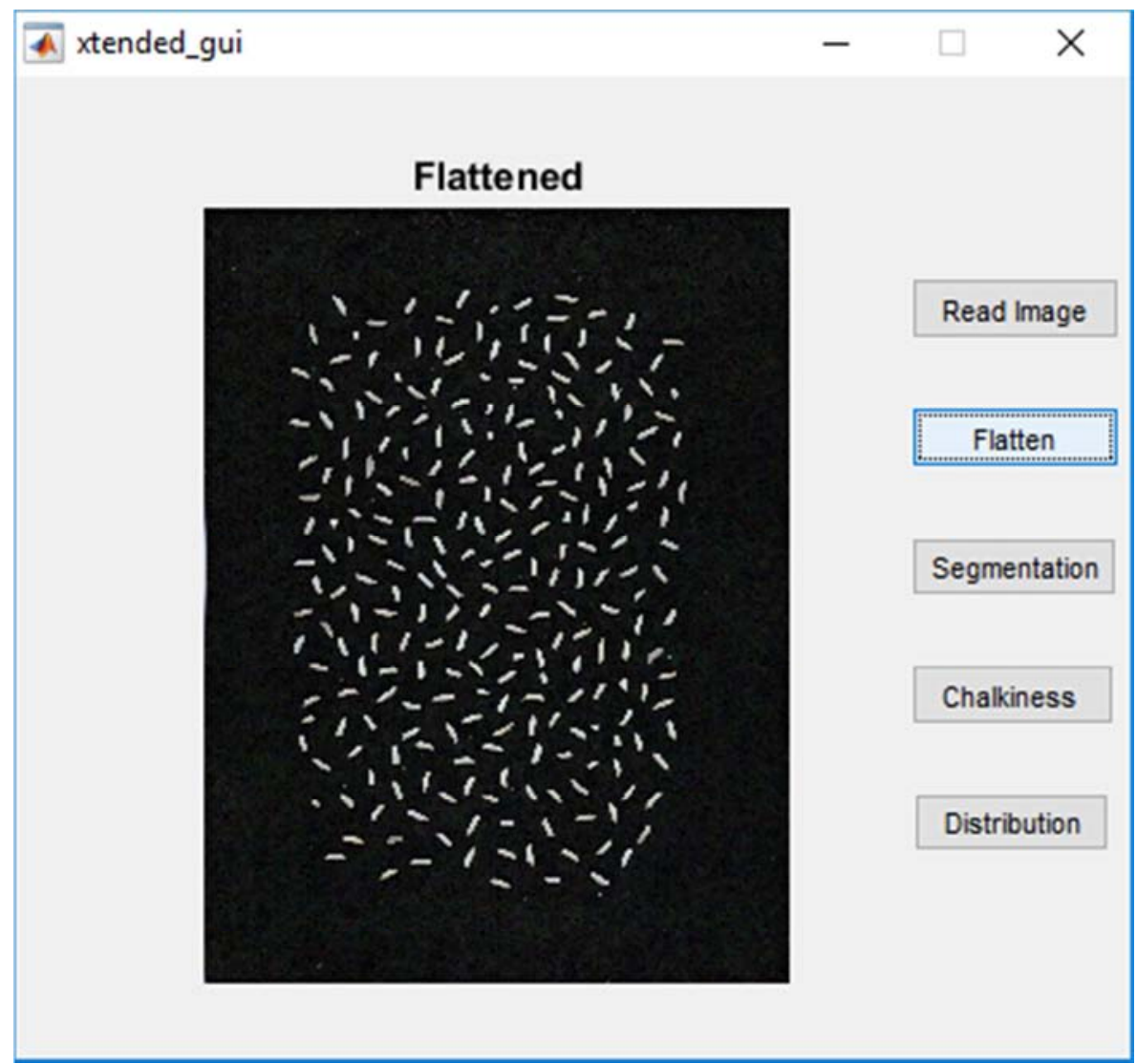

Figure 13. Screenshot Result of Flatten Condition for nurserySticky110.jpg.

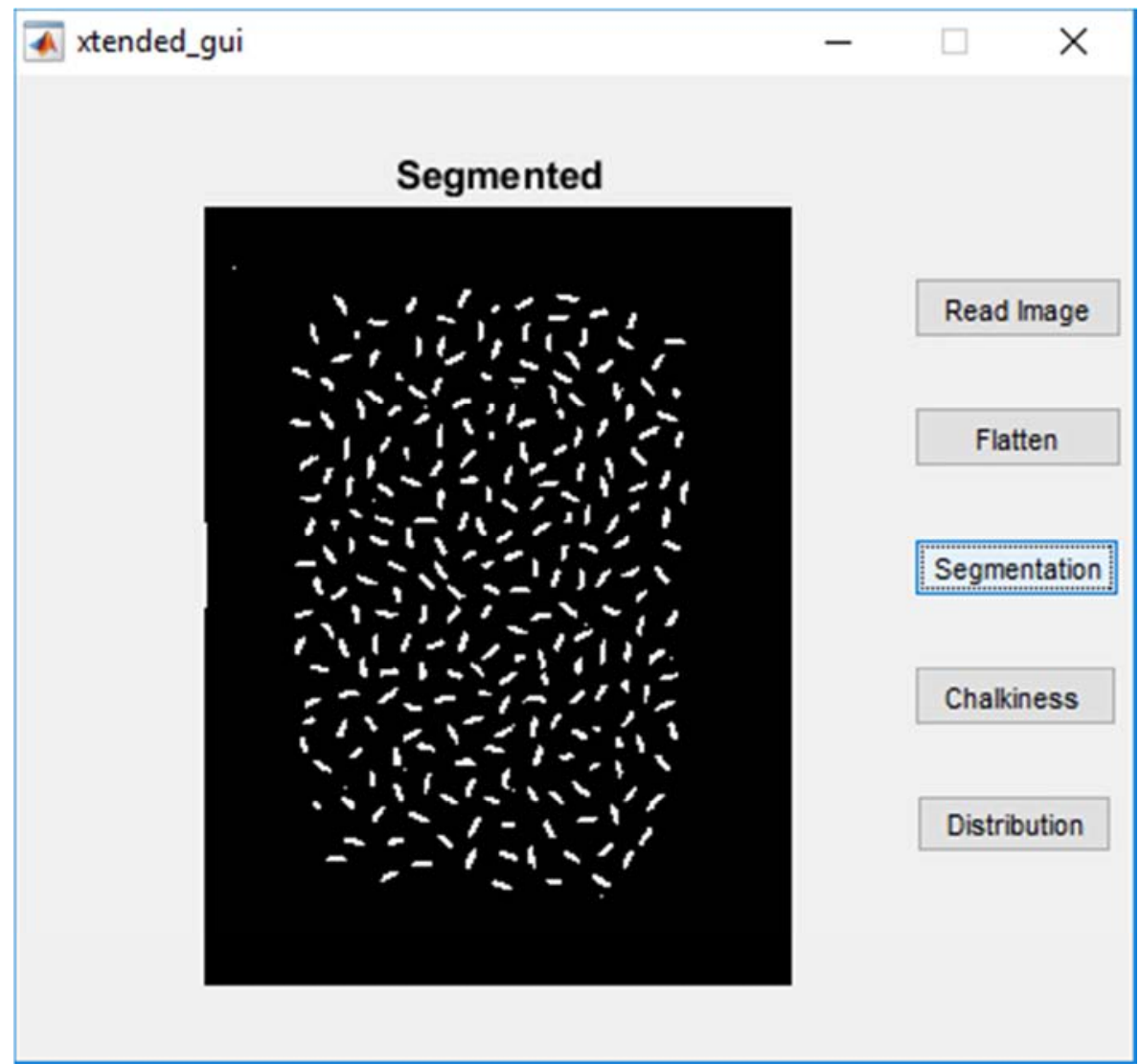

Figure 14. Screenshot Result of Threshold Condition for nurserySticky110.jpg. 


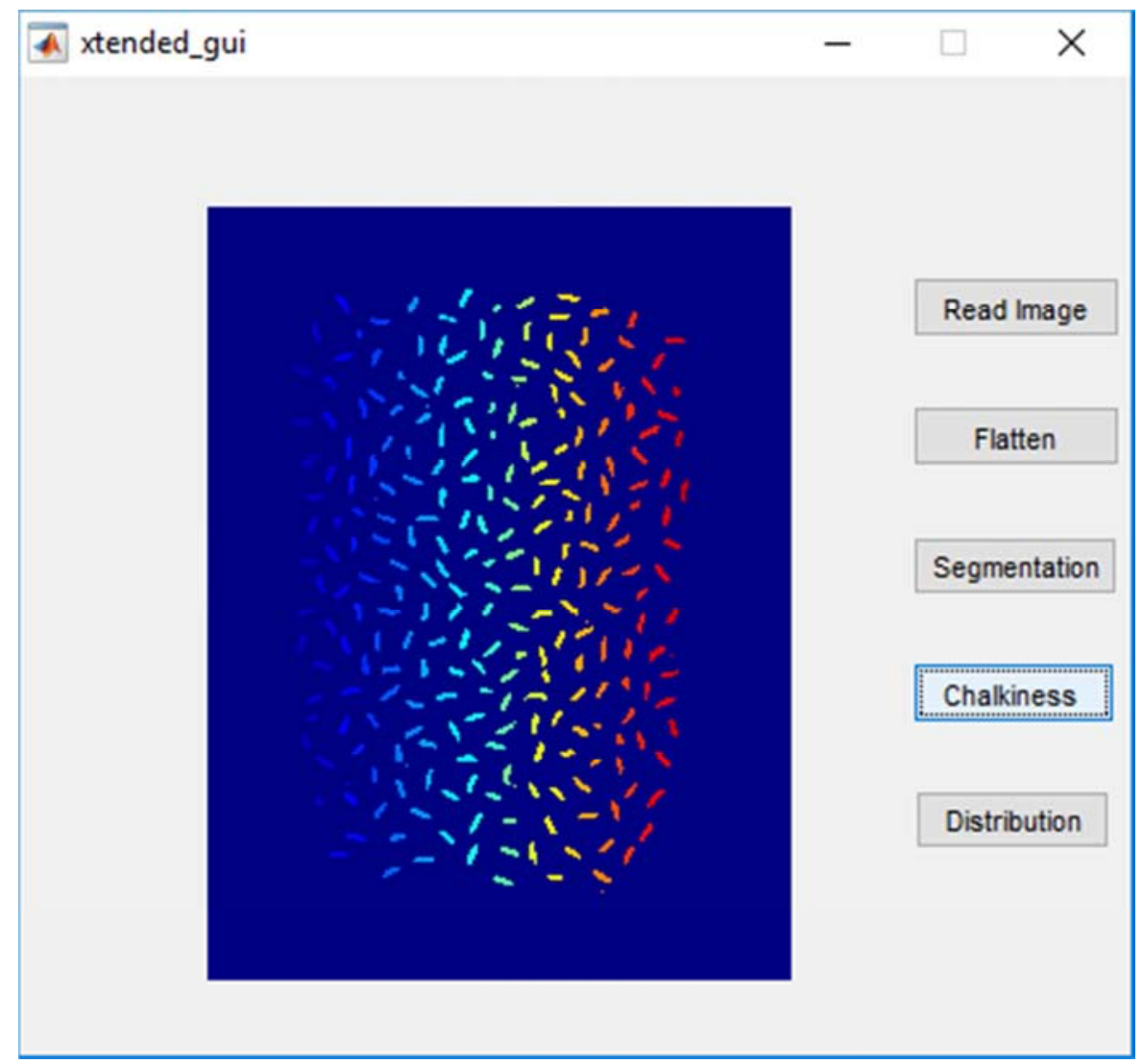

Figure 15. Screenshot Result of Chalkiness Detection for nurserySticky110.jpg.

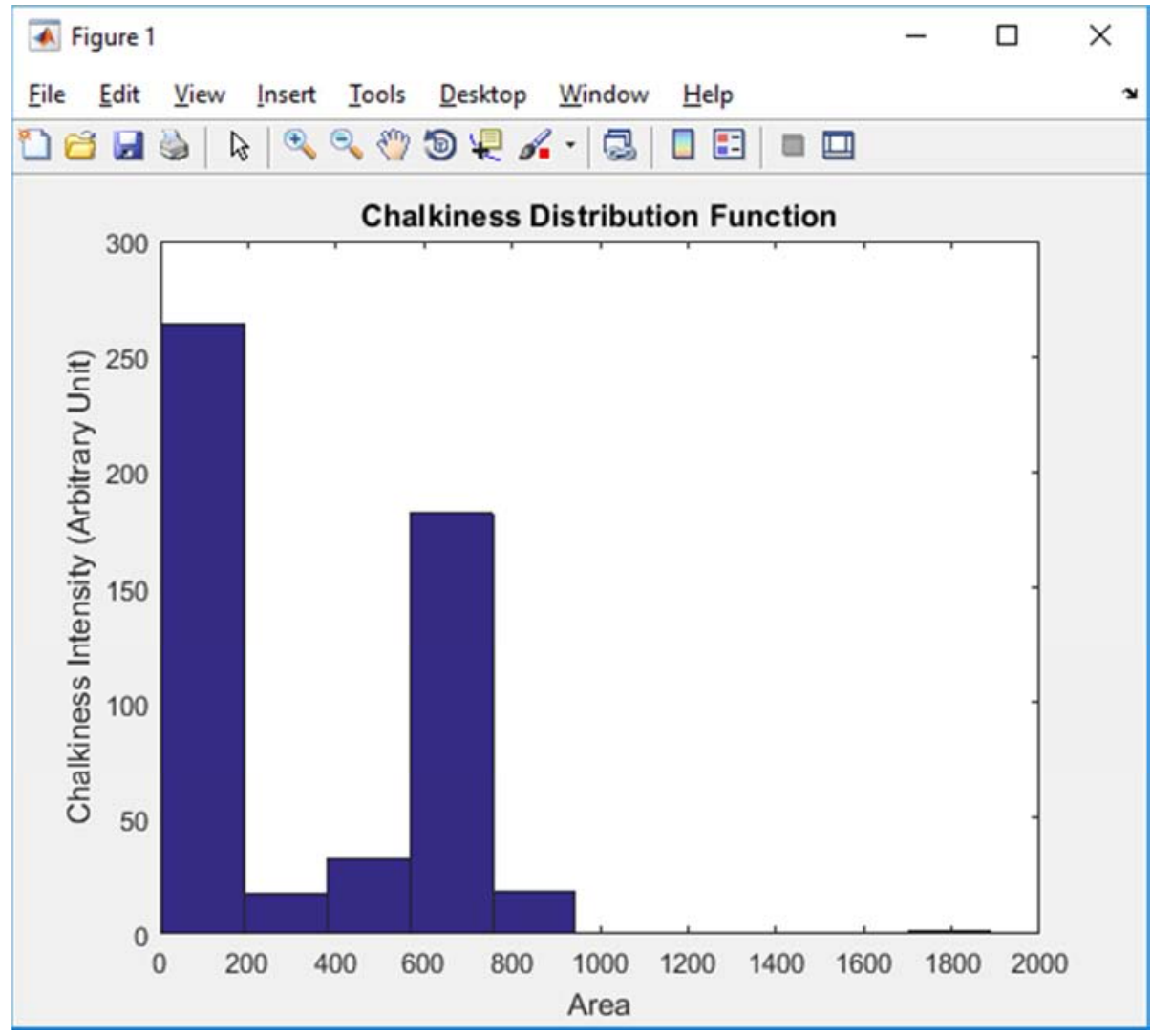

Figure 16. Chalkiness Distribution Function for nurserySticky110.jpg.

Figure 17 shows the screenshot result of read image of Paw-San C.jpg from the database. Figure 18 illustrates the 
screenshot result of flatten condition for Paw-San C.jpg. Figure 19 mentions the screenshot result of threshold condition for Paw-San C.jpg. Figure 20 illustrates the screenshot result of chalkiness detection for Paw-San C.jpg.
Figure 21 demonstrates the chalkiness distribution function for Paw-San C.jpg. According to the distribution function, the area of 0 to 500 is the highest level and the area of 500 to 1000 is the lowest level.

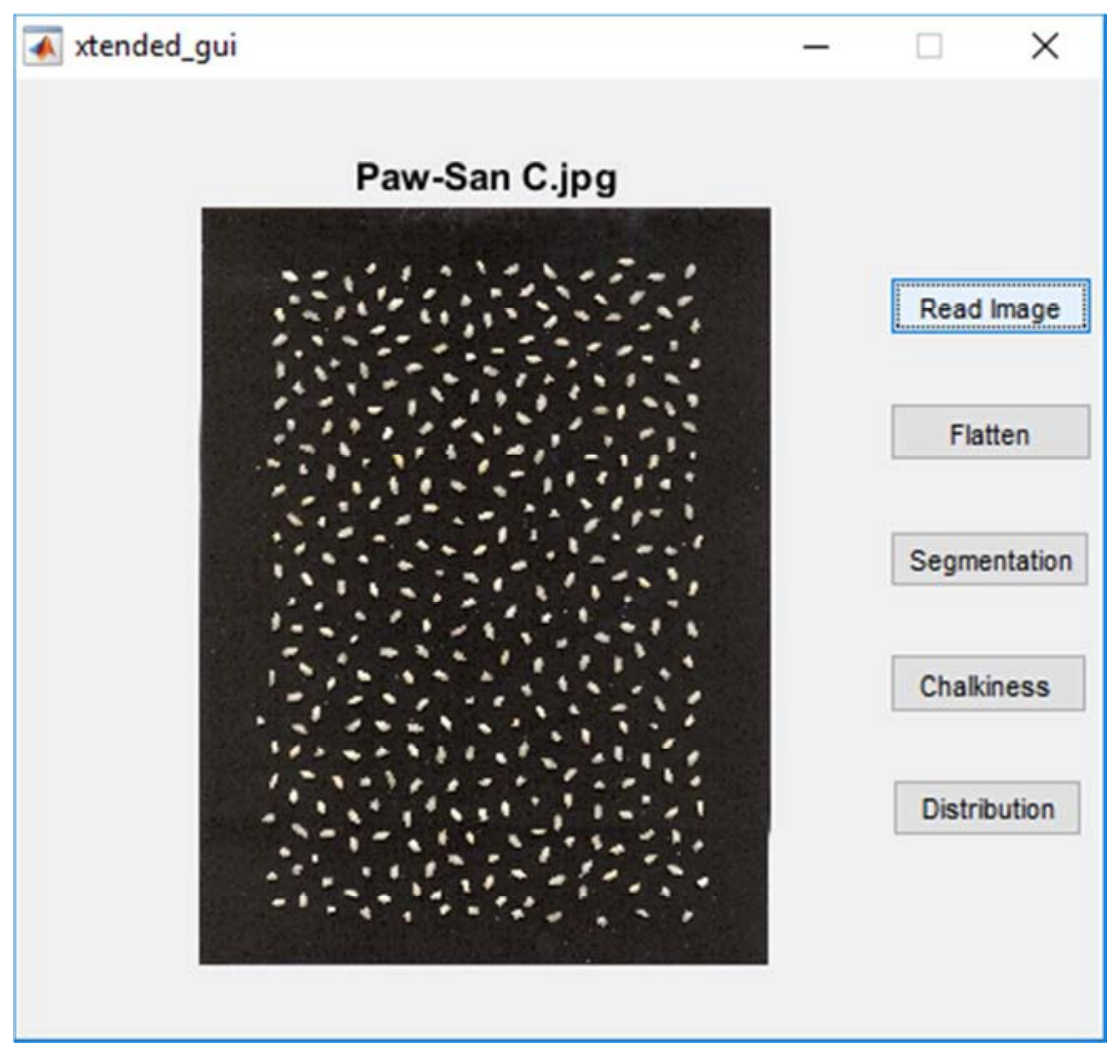

Figure 17. Screenshot Result of Read Image of Paw-San C.jpg from the Database.

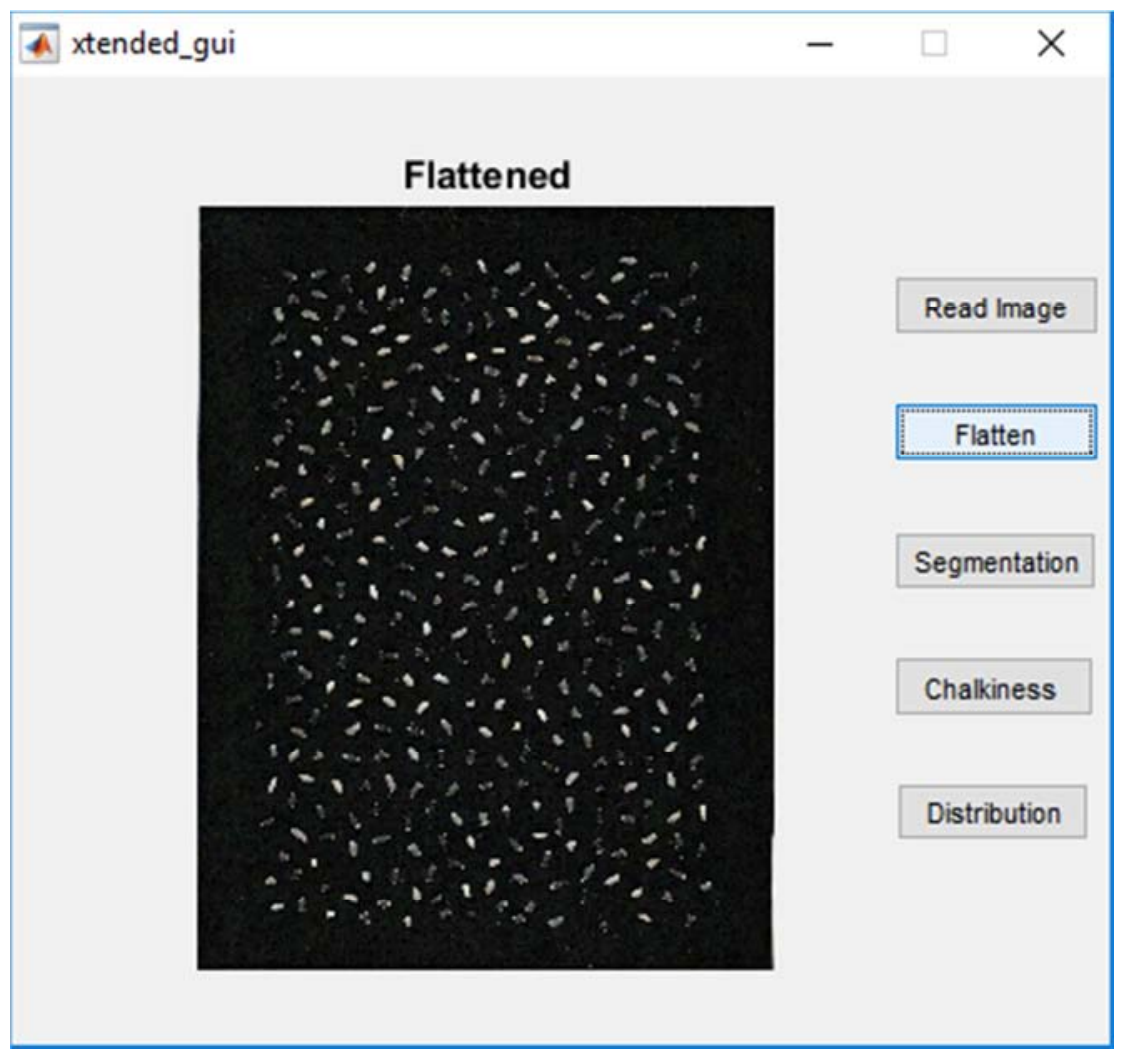

Figure 18. Screenshot Result of Flatten Condition for Paw-San C.jpg. 


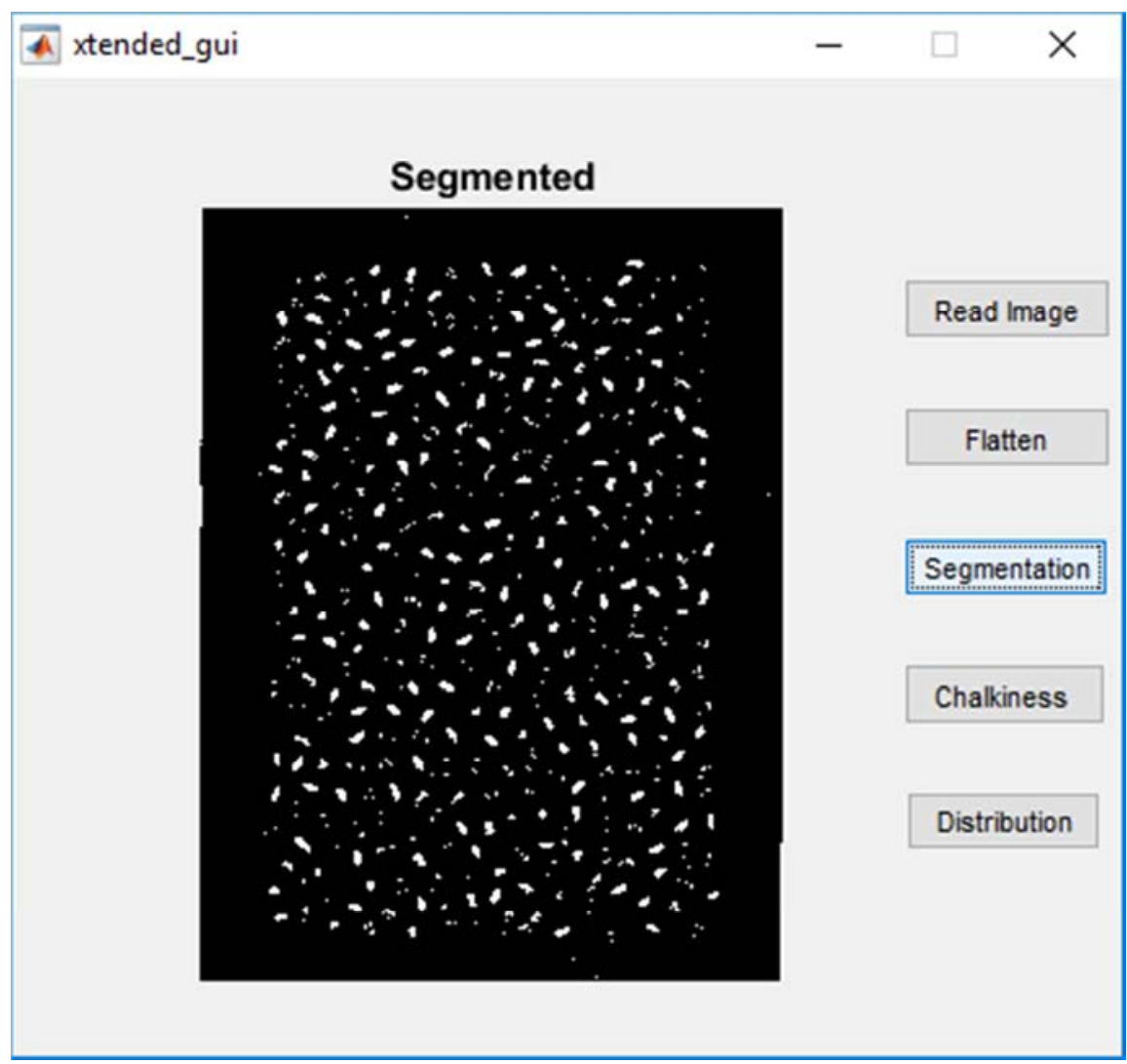

Figure 19. Screenshot Result of Threshold Condition for Paw-San C.jpg.

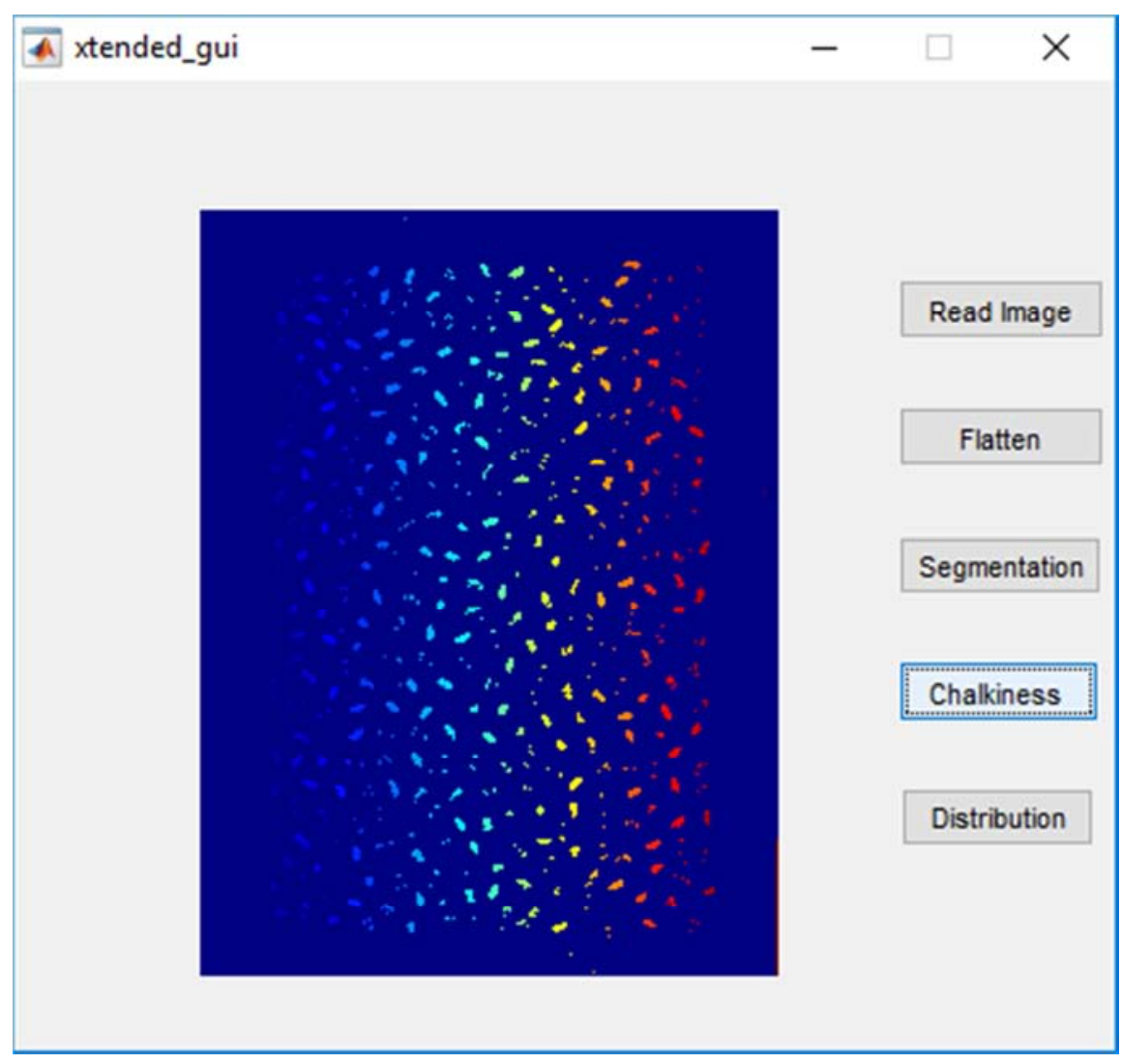

Figure 20. Screenshot Result of Chalkiness Detection for Paw-San C.jpg. 


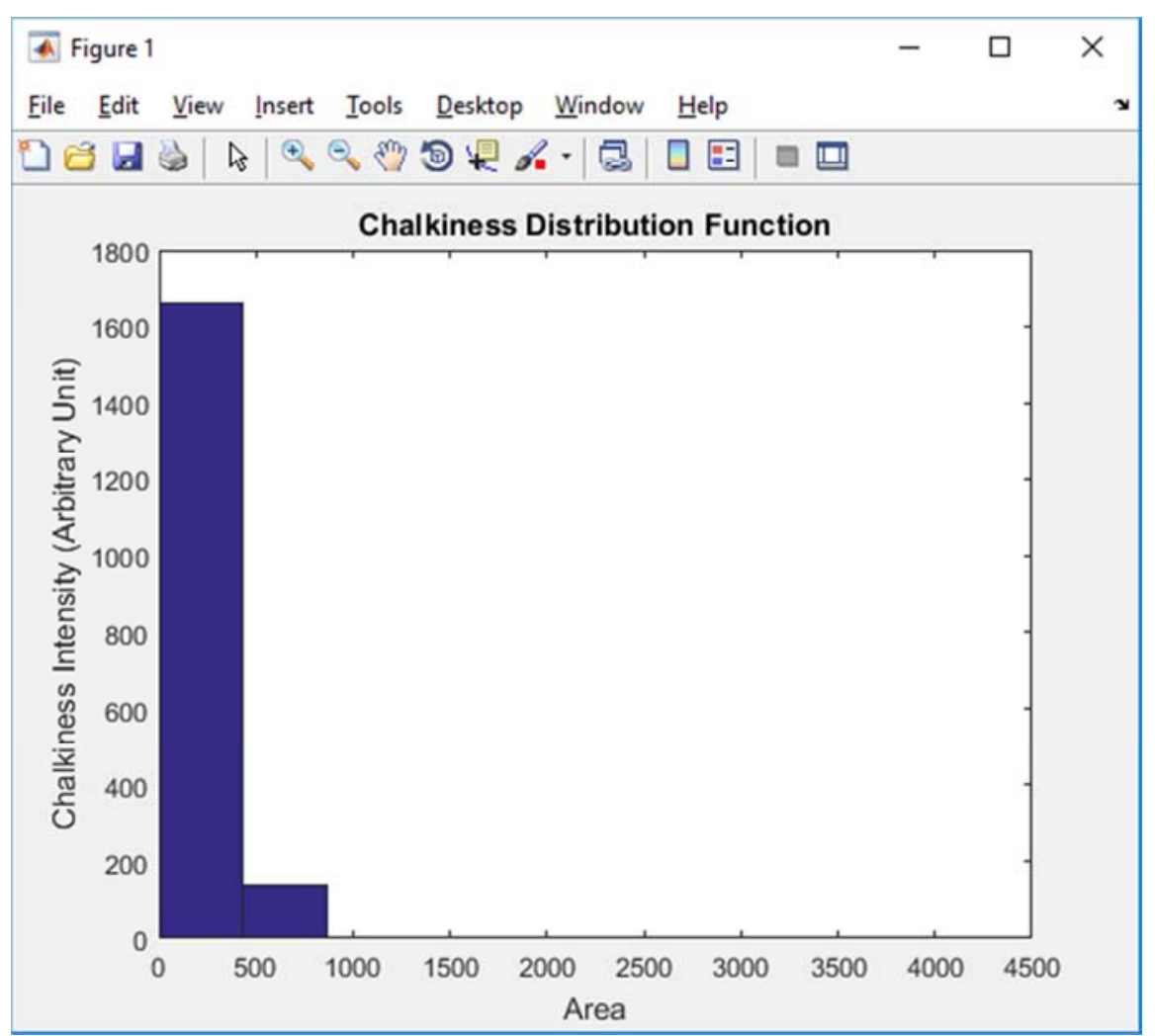

Figure 21. Chalkiness Distribution Function for Paw-San C.jpg.

Figure 22 illustrates the screenshot result of read image of zee yae10.jpg from the database. Figure 23 shows the screenshot result of flatten condition for zee yae10.jpg. Figure 24 mentions the screenshot result of threshold condition for zee yae10.jpg. Figure 25 points out the screenshot result of chalkiness detection for zee yae10.jpg. Figure 26 shows the chalkiness distribution function for zee yae10.jpg. According to the distribution function, the area of 0 to 100 is the highest level and the area of 200 to 900 is a Gaussian shape.

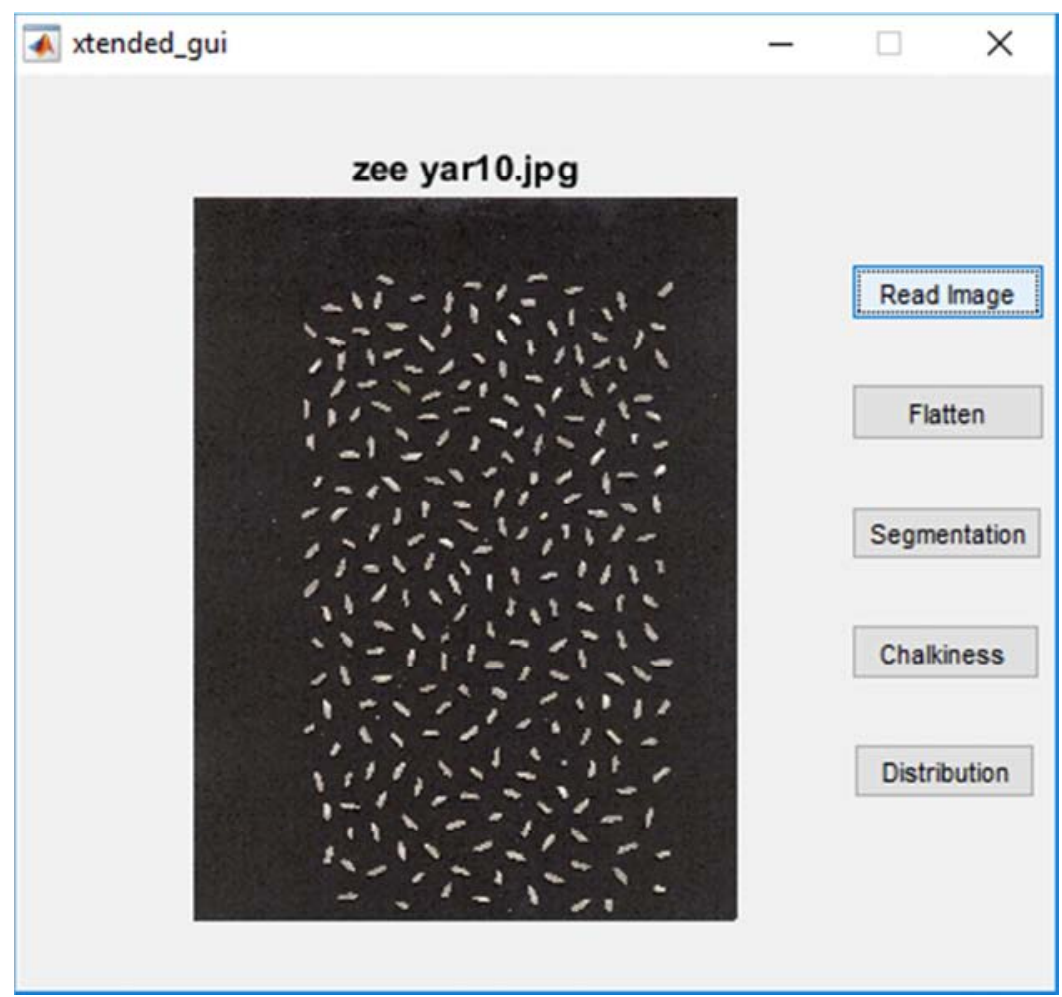

Figure 22. Screenshot Result of Read Image of zee yae10.jpg from the Database. 


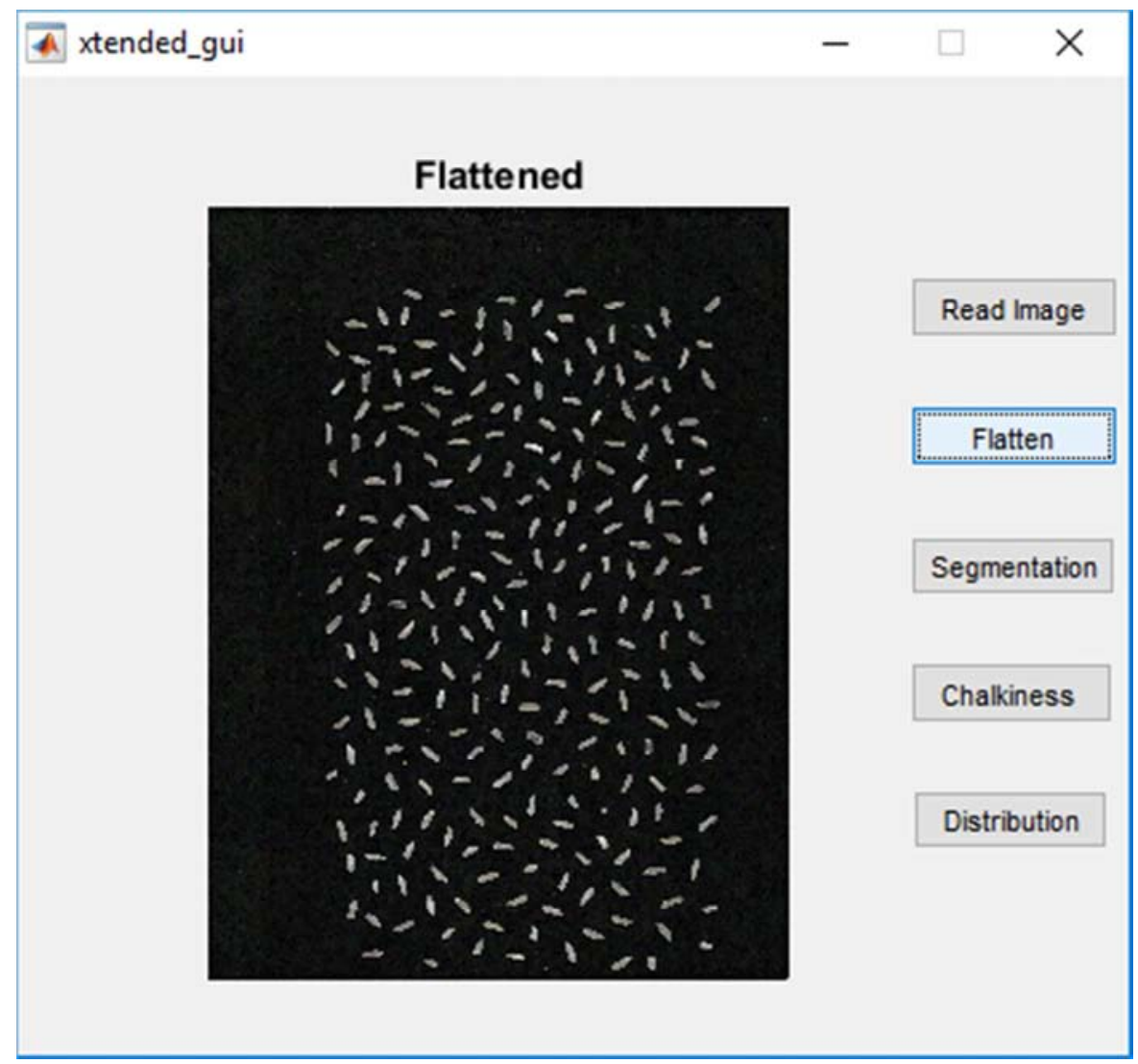

Figure 23. Screenshot Result of Flatten Condition for zee yae10.jpg.

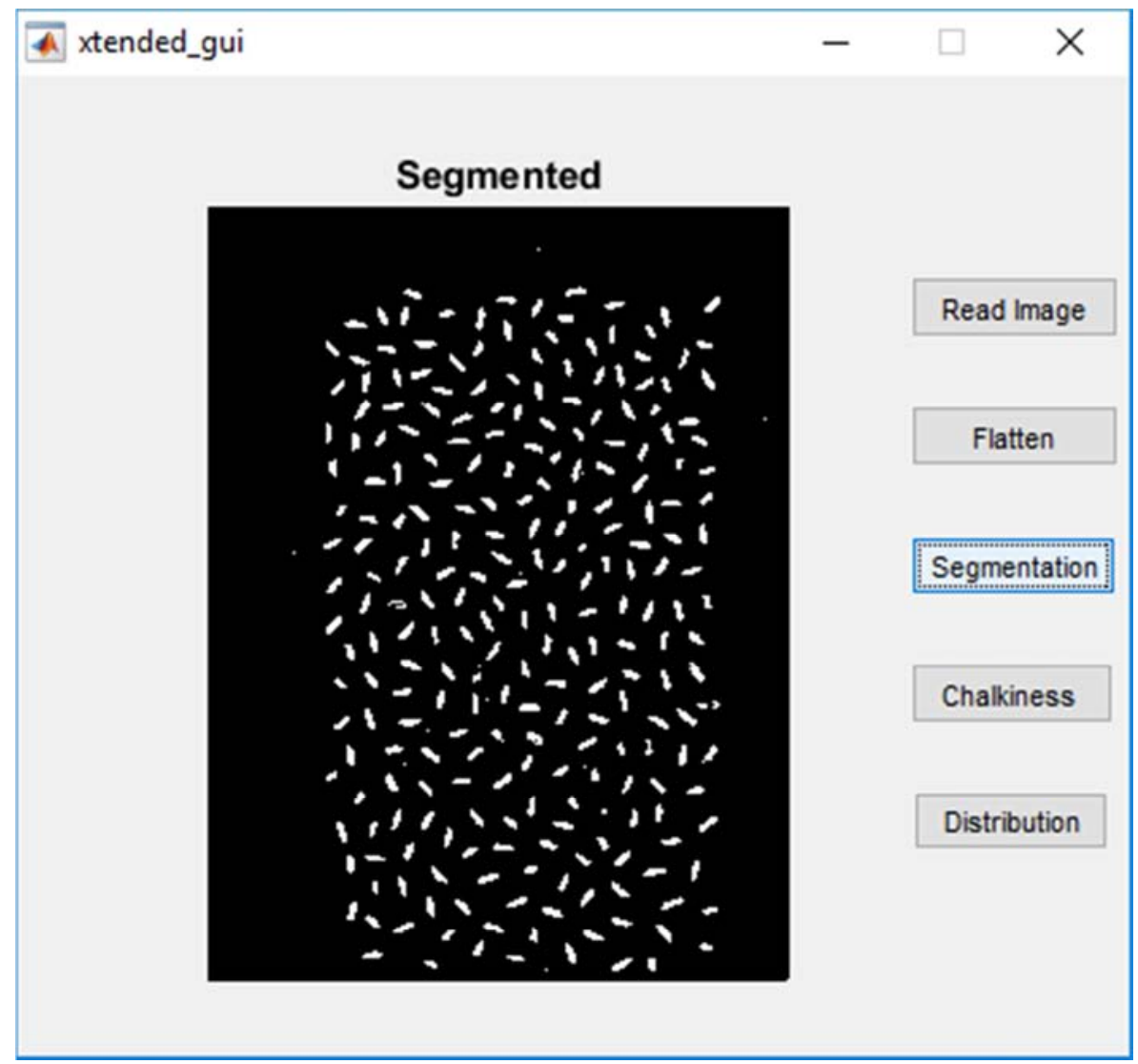

Figure 24. Screenshot Result of Threshold Condition for zee yae10.jpg. 


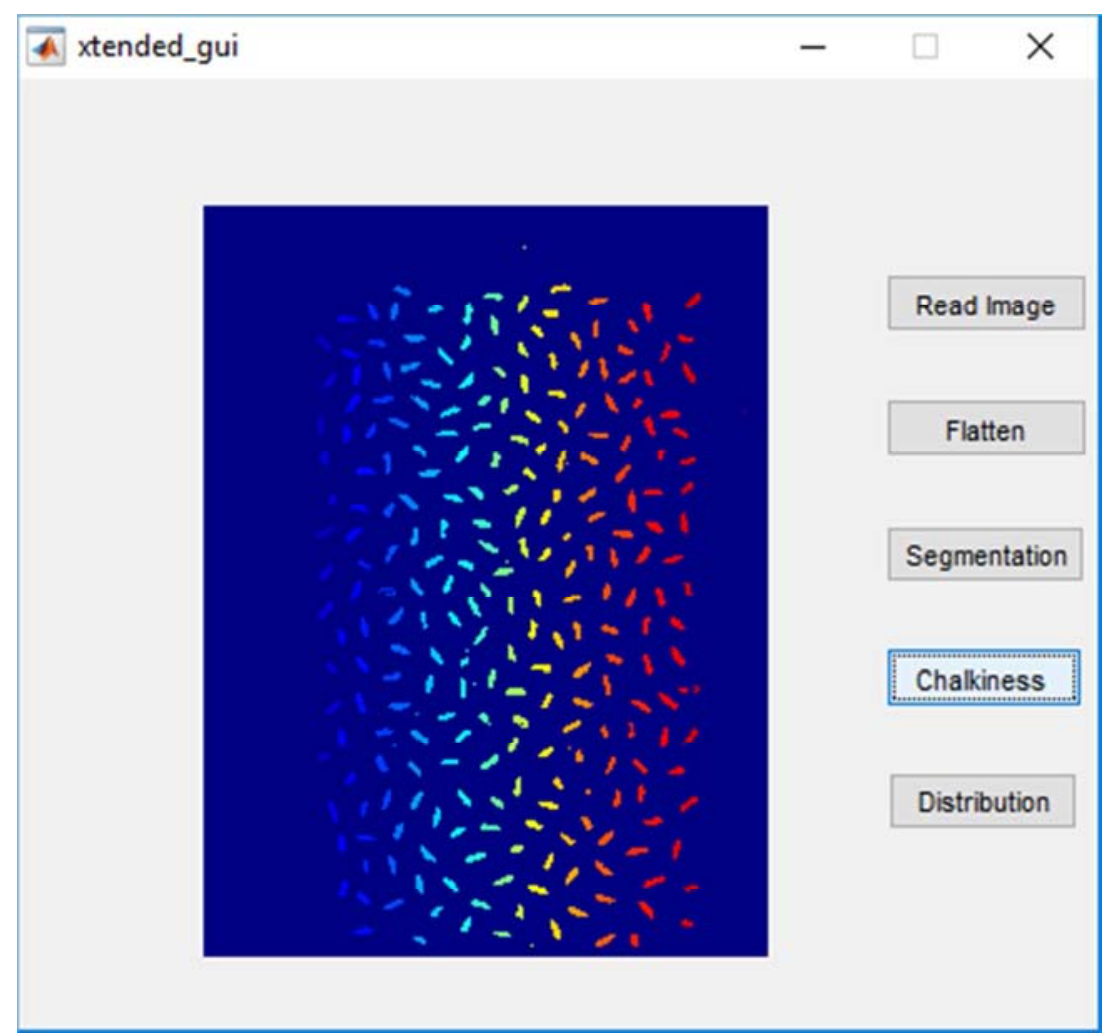

Figure 25. Screenshot Result of Chalkiness Detection for zee yae10.jpg.

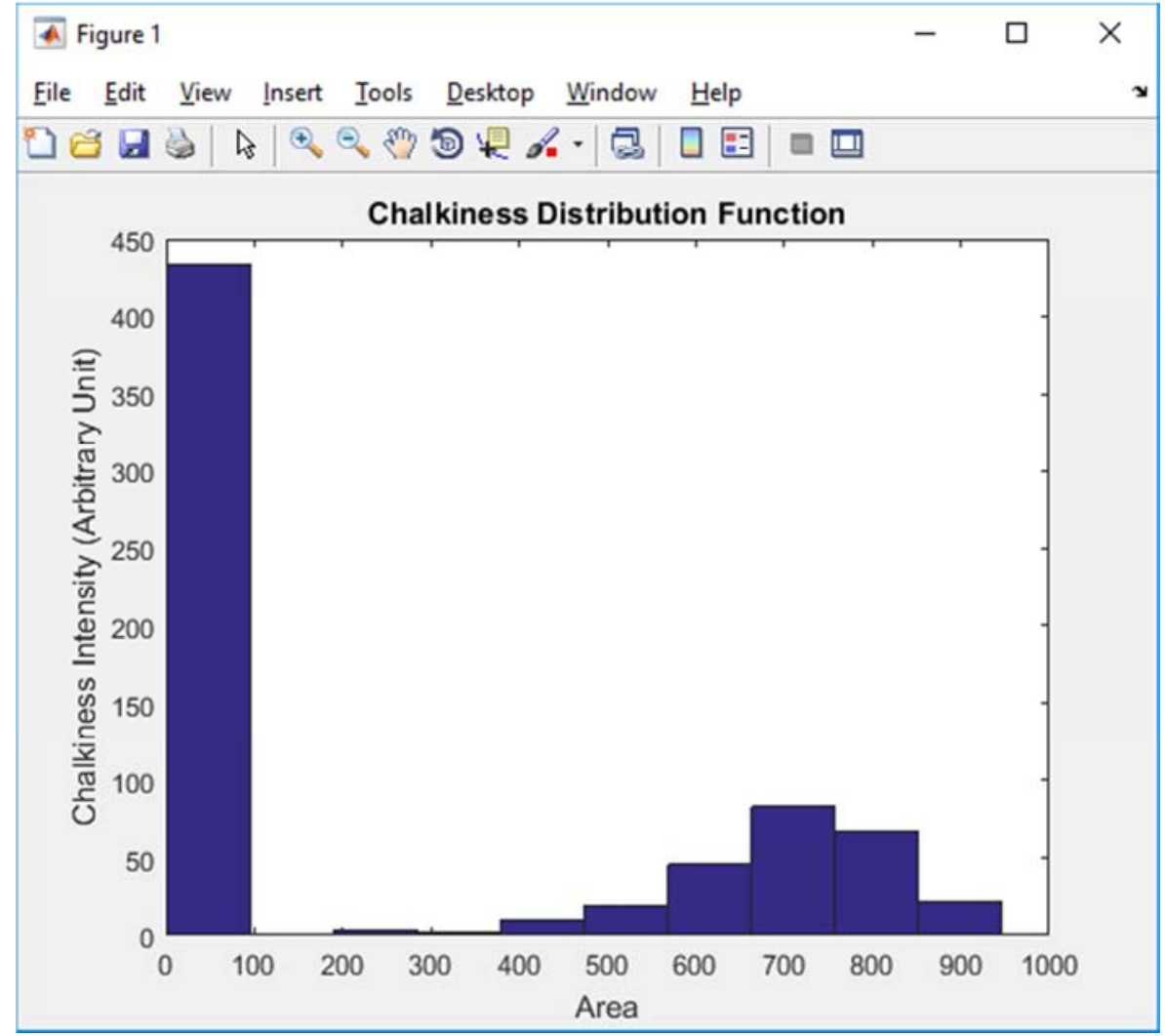

Figure 26. Chalkiness Distribution Function for zee yae10.jpg.

\section{Conclusion}

Rice chalkiness estimation is an important aspect of the agriculture research field. For the rice chalkiness estimation, rice chalkiness distribution function is one of its useful factors. In this paper a novel rice chalkiness detection method 
based on the real-time obtained rice images and the corresponding morphological processing is presented. The above method proves to be a very efficient way to determine the quality by analyzing the index value of the sample to be tested. MATLAB was used for programming the entire software for the experimentation. The setup used above is easily available. The results say that the detection method of chalkiness for Myanmar Rice met with the 90\% accuracy with computerize algorithm. The distribution function is one of the expressions for clearly mentioned for chalkiness level in the detection results. The Gaussian shape distribution points out the best detection level for Myanmar Rice. If the Gaussian shape could not be found in the detection level, the chalkiness level could not be easily detected. The distribution function mentions the area of rcie chalkiness for Myanmar rice. The method being more accurate than human eyes, fast and cost efficient and fit are to be used for industries.

\section{Acknowledgements}

The author would like to thank many contributors who participating in this works and especially my family who have supported many efforts to success this work. The contributions are supported by the Signal Processing Research group under the Department of Electronic Engineering of Yangon Technological University.

\section{References}

[1] Fitzgerald MA, McCouch SR, Hall RD: Not just a grain of rice: the quest for quality. Trends Plant Sci 2009, 14:133-139.

[2] Cheng SH, Zhuang JY, Fan YY, Du JH, Cao LY: Progress in research and development on hybrid rice: a super-domesticate in China. Ann Bot 2007, 100:959-966.

[3] Zhang QF: Strategies for developing green super rice. Proc Natl Acad Sci U S A 2007, 104:16402-16409.

[4] Wan XY, Weng JF, Zhai HQ, Wang JK, Lei CL, Liu XL, Guo T, Jiang L, Su N, Wan JM: Quantitative trait loci (QTL) analysis for rice grain width and fine mapping of an identified QTL allele gw-5 in a recombination hotspot region on Chromosome 5. Genetics 2008, 179:2239-2252.
[5] Yoshioka Y, Iwata H, Tabata M, Ninomiya S, Ohsawa R: Chalkiness in rice: potential for evaluation with image analysis. Crop Sci 2007, 47:2113-2120.

[6] Shen XP, Shen XY, Li G, Gon GP, Zhang HC: Effect of seeding time on chalkiness of liangyoupeijiu in Jiangsu rice growing areas at different latitudes. Chinese J Rice Sci 2007, 21:677-680.

[7] Tin HQ, Berg T, Bjørnstad Å: Diversity and adaptation in rice varieties under static (ex situ) and dynamic (in situ) management. Euphytica 2001, 122:491-502.

[8] Cheng FM, Zhong LJ, Wang F, Zhang GP: Differences in cooking and eating properties between chalky and translucent parts in rice grains. Food Chem 2005, 90:39-46.

[9] Wang JK, Wan XY, Li HH, Pfeiffer WH, Crouch J, Wan JM: Application of identified QTL-marker associations in rice quality improvement through a design-breeding approach. Theor Appl Genet 2007, 115:87-100.

[10] Liu X, Wan X, Ma X, Wan J: Dissecting the genetic basis for the effect of rice chalkiness, amylose content, protein content, and rapid viscosity analyzer profile characteristics on the eating quality of cooked rice using the chromosome segment substitution line population across eight environments. Genome 2011, 54:64-80.

[11] Zheng L, Zhang W, Liu S, Chen L, Liu X, Chen X, Ma J, Chen W, Zhao Z, Jiang L, Wan J: Genetic relationship between grain chalkiness, protein content, and paste viscosity properties in a backcross inbred population of rice. $\mathrm{J}$ Cereal Sci 2012, 56:153-160.

[12] Lisle, A. J., M. Martin, and M. A. Fitzgerald. 2000. Chalky and translucent rice grains differ in starch composition and structure and cooking properties. Cereal Chem. 77:627-632.

[13] Kadan, R. S., R. J. Bryant, and J. A. Miller. 2008. Effects of milling on functional properties of rice flour. J. Food Sci. 73:E151-E154.

[14] Cooper, N. T. W., T. J. Siebenmorgen, and P. A. Counce. 2008. Effects of nighttime temperature during kernel development on rice physicochemical properties. Cereal Chem. 85:276-282.

[15] J. Hemming and T. Rath, "PA-Precision Agriculture," Journal of Agricultural Engineering Research 78, 233-243 (2001). 